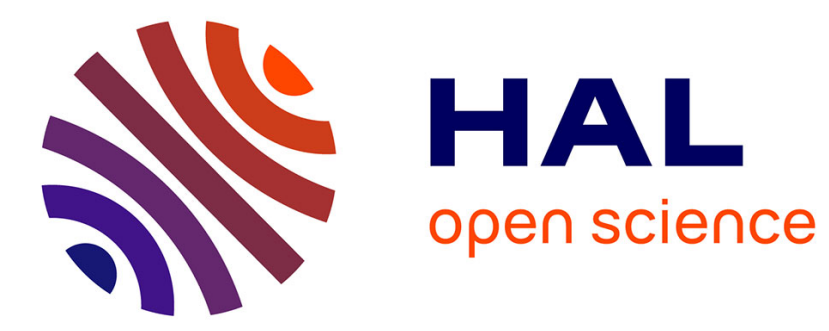

\title{
Temperley-Lieb Algebras At Roots of Unity, A Fusion category and the Jones Quotient
}

\author{
Kenji Iohara, Gus I. Lehrer, Ruibin B Zhang
}

\section{To cite this version:}

Kenji Iohara, Gus I. Lehrer, Ruibin B Zhang. Temperley-Lieb Algebras At Roots of Unity, A Fusion category and the Jones Quotient. Mathematical Research Letters, 2019, 26 (1), pp.121-158. 10.4310/mrl.2019.v26.n1.a8 . hal-01476831v3

\section{HAL Id: hal-01476831 \\ https://hal.science/hal-01476831v3}

Submitted on 29 Dec 2017

HAL is a multi-disciplinary open access archive for the deposit and dissemination of scientific research documents, whether they are published or not. The documents may come from teaching and research institutions in France or abroad, or from public or private research centers.
L'archive ouverte pluridisciplinaire HAL, est destinée au dépôt et à la diffusion de documents scientifiques de niveau recherche, publiés ou non, émanant des établissements d'enseignement et de recherche français ou étrangers, des laboratoires publics ou privés. 


\title{
TEMPERLEY-LIEB ALGEBRAS AT ROOTS OF UNITY, A FUSION CATEGORY AND THE JONES QUOTIENT.
}

\author{
K. IOHARA, G.I. LEHRER AND R.B. ZHANG
}

\begin{abstract}
When the parameter $q$ is a root of unity, the Temperley-Lieb algebra $\mathrm{TL}_{n}(q)$ is non-semisimple for almost all $n$. In this work, using cellular methods, we give explicit generating functions for the dimensions of all the simple $\mathrm{TL}_{n}(q)$ modules. Jones showed that if the order $\left|q^{2}\right|=\ell$ there is a canonical symmetric bilinear form on $\mathrm{TL}_{n}(q)$, whose radical $R_{n}(q)$ is generated by a certain idempotent $E_{\ell-1} \in \mathrm{TL}_{\ell-1}(q) \subseteq \mathrm{TL}_{n}(q)$, which is now referred to as the Jones-Wenzl idempotent, for which an explicit formula was subsequently given by Graham and Lehrer. Although the algebras $Q_{n}(\ell):=\mathrm{TL}_{n}(q) / R_{n}(q)$, which we refer to as the Jones algebras (or quotients), are not the largest semisimple quotients of the $\mathrm{TL}_{n}(q)$, our results include dimension formulae for all the simple $Q_{n}(\ell)$-modules. This work could therefore be thought of as generalising that of Jones et al. on the algebras $Q_{n}(\ell)$. We also treat a fusion category $\mathcal{C}_{\text {red }}$ introduced by Reshitikhin, Turaev and Andersen, whose simple objects are the quantum $\mathfrak{s l}_{2}$-tilting modules with non-zero quantum dimension, and which has an associative truncated tensor product referred to below as the fusion product. We show $Q_{n}(\ell)$ is the endomorphism algebra of a certain module in $\mathcal{C}_{\text {red }}$ and use this fact to recover a dimension formula for $Q_{n}(\ell)$. We also show how to construct a "stable limit" $K\left(Q_{\infty}\right)$ of the corresponding fusion category of the $Q_{n}(\ell)$, whose structure is determined by the fusion rule of $\mathcal{C}_{\text {red }}$, and observe a connection with a fusion category of affine $\mathfrak{s l}_{2}$.
\end{abstract}

\section{INTRODUCTION}

The Temperley-Lieb algebras $\mathrm{TL}_{n}(q)$ (see $\S 4$ below) are algebras over a ring $R$ which depend on a parameter $q \in R$. They occur in many areas of mathematics and physics, and may be characterised as the endomorphism algebras of the objects in the Temperley-Lieb category (see [13]). In this work we shall generally take $R=\mathbb{C}$. These algebras are well known to have a cellular structure [12] and their representation theory may be analysed using this structure.

For generic vaues of $q$, the algebra $\mathrm{TL}_{n}(q)$ is semisimple, and its simple modules are the cell modules $W_{t}(n)$, for $t \in \mathbb{Z}, 0 \leq t \leq n$ and $t \equiv n(\bmod 2)$. However when $q$ is a root of unity, the cell modules are often no longer simple, but have a simple head $L_{t}(n)$. The modules $L_{t}(n)$, where $t$ runs over the same values as above, form a complete set of simple modules for $\mathrm{TL}_{n}(q)$ in this case.

In this work, our first purpose is to give explicit formulae for the dimensions of the modules $L_{t}(n)$. This will be done by deriving, for each $t \in \mathbb{Z}_{\geq 0}$, an explicit formula for the generating function

2010 Mathematics Subject Classification. 81R15, 16W22, 46L37 .

The present work was initiated during an Australian Research Council funded visit of K. I. to the University of Sydney in October-November 2016. He gratefully acknowledges the support and hospitality extended to him. 


$$
L_{t}(x):=\sum_{k=0}^{\infty} \operatorname{dim}\left(L_{t}(t+2 k)\right) x^{k} .
$$

The algebra $\mathrm{TL}_{n}(q)$ has a trace $\operatorname{tr}_{n}: \mathrm{TL}_{n}(q) \rightarrow \mathbb{C}$, identified by Jones, whose associated bilinear form is generically non-degenerate (see (2.2) below). If $q$ is a root of unity, and the order $\left|q^{2}\right|=\ell$, then $\operatorname{tr}_{n}$ has a radical of dimension 1 if $n=\ell-1$, the generating element being the Jones-Wenzl idempotent $E_{\ell-1} \in \mathrm{TL}_{\ell-1}(q)$. An explicit formula for $E_{\ell-1}$ is given in [13]. Jones has shown [18, Thm. 2.1] that in this case, for any $n \geq \ell-1$, the radical $R_{n}(q)$ of $\operatorname{tr}_{n}$ is generated by $E_{\ell-1} \in \mathrm{TL}_{\ell-1}(q) \subseteq \mathrm{TL}_{n}(q)$. Moreover, for $n \geq \ell$, the algebra $\mathrm{TL}_{n}(q)$ has the canonical semisimple quotient $Q_{n}(\ell):=\mathrm{TL}_{n}(q) / R_{n}(q)$, which we refer to as the Jones algebra.

As a consequence of our analysis, we deduce a complete description of the simple representations of the Jones algebras $Q_{n}(\ell)$, as well as a generating function for its dimension, which recovers a result of [11]. Note that $Q_{n}(q)$ is far from being the maximal semisimple quotient of $\mathrm{TL}_{n}(q)$, as our work shows.

In $\S 8$ we relate $Q_{n}(\ell)$ to the fusion category $\mathcal{C}_{\text {red }}$ introduced by Reshetikhin, Turaev and Andersen [25, 1] whose objects are sums of the indecomposable tilting modules of non-zero quantum dimension for the quantum group $\mathrm{U}_{q}\left(\mathfrak{s l}_{2}\right)$, when $q^{2}$ is a primitive $\ell^{\text {th }}$ root of unity. The category $\mathcal{C}_{\text {red }}$ has a fusion product $\underline{\otimes}$, and if $\Delta_{q}(1)$ is the indecomposable (in fact simple) tilting module with highest weight 1 , we show that $Q_{n}(\ell) \cong \operatorname{End}_{\mathrm{U}_{q}\left(\mathfrak{s l}_{2}\right)}\left(\Delta_{q}(1)^{\underline{\otimes}}\right)$. Together with our earlier results, this recovers a formula for the dimension of $Q_{n}(\ell)$ due to Jones [11].

We note also that our results are related to those of [5], which could be thought of as treating the more complicated positive characteristic analogue of some of our material.

Acknowledgement The authors would like to thank the referee for a very thorough reading of the manuscript, and in particular for detecting an error in the original version. The paper has benefited significantly from the referee's input.

\section{The Temperley-Lieb Algebras.}

2.1. Definitions. In this work, all algebras will be over $\mathbb{C}$. Much of the theory we develop applies over more general domains, but since we will be concerned here with connections to the theory of operator algebras and mathematical physics, we limit our discussion to $\mathbb{C}$-algebras. For $n \in \mathbb{N}$, the Temperley-Lieb algebra $\mathrm{TL}_{n}(q)$ is defined as follows.

Definition 2.1. Let $q \in \mathbb{C}$. $\mathrm{TL}_{n}=\mathrm{TL}_{n}(q)$ is the associative $\mathbb{C}$-algebra with generators $f_{1}, f_{2}, \ldots, f_{n-1}$ and relations

$$
\begin{aligned}
f_{i}^{2} & =-\left(q+q^{-1}\right) f_{i} \text { for all } i \\
f_{i} f_{i \pm 1} f_{i} & =f_{i} \text { for all } i \\
f_{i} f_{j} & =f_{j} f_{i} \text { if }|i-j| \geq 2 .
\end{aligned}
$$

2.2. The Jones form. In his seminal work [15] on subfactors of a factor, Jones showed that certain projectors $\left\{e_{1}, \ldots, e_{n-1}\right\}(n=1,2,3, \ldots)$ in a von Neumann algebra satisfy the Temperley-Lieb-like relations, a fact that led to the definition of the "Jones polynomial" of an oriented link. In the notation of $[17$, p. 104, (I)-(VI)], 
Jones showed that if $f_{i}=\left(q+q^{-1}\right) e_{i}$, then the $f_{i}$ satisfy the relations (2.1), where Jones' parameter $t$ is replaced by $q^{2}$. If $q^{2} \neq-1$, Jones' form on $\mathrm{TL}_{n}(q)$ is defined as the unique (invariant) $\operatorname{trace} \operatorname{tr}_{n}=\operatorname{tr}$ on $\mathrm{TL}_{n}(q)$ which satisfies

$$
\operatorname{tr}(1)=1 \text { and } \operatorname{tr}\left(x f_{i}\right)=-\left(q+q^{-1}\right)^{-1} \operatorname{tr}(x) \text { for } x \in \mathrm{TL}_{i-1} \subset \mathrm{TL}_{i} \subseteq \mathrm{TL}_{n}
$$

for $1 \leq i \leq n-1$.

This trace on $\mathrm{TL}_{n}(q)$ is non-degenerate if and only if $q^{2}$ is not a root of unity, or, if $\left|q^{2}\right|=\ell, n \leq \ell-2\left([13,(3.8)]\right.$. Thus the discrete set of values of $q^{2}$ for which Jones' sequence $\left(A_{n}\right)$ of algebras is infinite coincides precisely with the set of values of $q^{2}$ for which the trace form above on $\operatorname{TL}_{n}(q)$ is degenerate.

2.3. Cell modules and forms. Let us fix $n$ and consider the representation theory of $\mathrm{TL}_{n}$.

Recall [13] that the Temperley-Lieb category $\mathbf{T}$ over $\mathbb{C}$ has object set $\mathbb{N}$ and for $t, n \in \mathbb{N}, \operatorname{Hom}_{\mathbf{T}}(t, n)$ is the vector space with basis the set of Temperley-Lieb diagrams from $t$ to $n$, i.e., which have $t$ lower vertices, and $n$ upper vertices. Composition is by concatenation of diagrams, with free circles replaced by $-\left(q+q^{-1}\right)$. In speaking of Temperley-Lieb diagrams, we shall freely use the well known terminology which refers to upper arcs (which join two upper vertices), lower arcs (which join two lower vertices) and through strings (which join an upper vertex to a lower vertex).

A diagram from $t$ to $n$ is monic if it has a left inverse. This means (assuming $\left.q+q^{-1} \neq 0\right)$ that there are no lower arcs. The algebra $\operatorname{Hom}_{\mathbf{T}}(n, n)$ is the TemperleyLieb algebra $\mathrm{TL}_{n}=\mathrm{TL}_{n}(q)$.

By [12] $\mathrm{TL}_{n}$ has cell modules $W_{t}:=W_{t}(n)$ whose basis is the set of monic Temperley-Lieb morphisms from $t$ to $n$, where $t \in \mathcal{T}(n)$, and $\mathcal{T}(n)=\{t \in \mathbb{Z} \mid$ $0 \leq t \leq n$ and $t+n \in 2 \mathbb{Z}\}$.

Now $W_{t}$ has an invariant form $(,)_{t}$ which may be described as follows. For monic diagrams $D_{1}, D_{2}: t \rightarrow n$, we form the diagram $D_{1}^{*} D_{2}: t \rightarrow t$. If $D_{1}^{*} D_{2}$ is monic (i.e. a multiple of $\mathrm{id}_{t}$ ), then we write $D_{1}^{*} D_{2}=\left(D_{1}, D_{2}\right) \mathrm{id}_{t}$; otherwise we say $\left(D_{1}, D_{2}\right)=0$. Here $D^{*}$ denotes the diagram obtained from $D$ by reflection in a horizontal, extended to $W_{t}$ by linearity.

The form $(,)_{t}$ is evidently equivariant for the $\mathrm{TL}_{n}(q)$-action; That is, we have for any element $a \in \mathrm{TL}_{n}(q)$ and elements $v, w \in W_{t}(n),(a v, w)_{t}=\left(v, a^{*} w\right)_{t}$. Hence the radical $\operatorname{Rad}_{t}$ of the form $(,)_{t}$ is a submodule of $W_{t}$. Let $L_{t}:=W_{t} / \operatorname{Rad}_{t}$. The general theory asserts that the $L_{t}$ are simple, and represent all the distinct isomorphism classes of simple $\mathrm{TL}_{n}(q)$-modules.

Remark 2.2 (Notation). Note that $W_{t}, \operatorname{Rad}_{t}$ and $L_{t}$ may be regarded as functors $: \mathbf{T} \rightarrow$ vect, where vect is the category of finite dimensional $\mathbb{C}$-vector spaces. Thus $W_{t}(n)$ is the evaluation of the functor at $n$. However, when the context makes it clear, we shall abuse notation by writing $W_{t}$ for $W_{t}(n)$, etc.

\section{SEMISIMPLICITY AND NON-DEGENERACY.}

Clearly, if the trace (2.2) is non-degenerate, the algebra $\mathrm{TL}_{n}$ is semisimple. The converse is true except for one single case (see [13, Rem. 3.8, p.204]). It follows from [13, Cor. (3.6)] that if $\left|q^{2}\right|=\ell, \mathrm{TL}_{n}$ is non-semisimple if and only if $n \geq \ell$. 
Moreover we have very precise information concerning the radical of the invariant trace form.

3.1. Radical of the trace form. The radical of the trace form above is given by the following result (see $[13, \S 3],[16]$ ).

Proposition 3.1. ([18, Thm. 2.1]) If $q$ is not a root of unity then $\operatorname{tr}_{n}$ is nondegenerate and $\mathrm{TL}_{n}$ is semisimple for all $n$.

Suppose the order of $q^{2}$ is $\ell$. Then there is a unique idempotent $E_{\ell-1} \in \mathrm{TL}_{\ell-1}$ (the Jones-Wenzl idempotent) such that $f_{i} E_{\ell-1}=E_{\ell-1} f_{i}=0$ for $1 \leq i \leq \ell-2$. Moreover for $n \geq \ell-1$ the radical of $\operatorname{tr}_{n}$ is generated as ideal of $\mathrm{TL}_{n}$ by $E_{\ell-1}$.

Remark 3.2. cf. [13, Remark (3.8)] It follows from Proposition 3.1 that the trace $\operatorname{tr}_{n}$ is non-degenerate if and only if $n \leq \ell-2$, where $\ell=\left|q^{2}\right|$. It follows that the case $n=\ell-1$ is uniquely characterised as the one where the form $\operatorname{tr}_{n}$ is degenerate, but $\mathrm{TL}_{\ell-1}$ is semisimple.

Note also that $E_{\ell-1}$ is regarded as an element of $\mathrm{TL}_{n}$ for $n \geq \ell-1$ in the usual way, by thinking of it as $E_{\ell-1} \otimes I^{\otimes n-\ell+1}$.

The following formula for the idempotent $E_{\ell-1}$ was proved in [13, Cor. 3.7]. To prepare for its statement, recall that if $F$ is a finite forest (i.e. a partially ordered set in which $x \leq a, x \leq b \Longrightarrow a \leq b$ or $b \leq a)$, then we define a Laurent polynomial

$$
h_{F}(x)=\frac{[|F|]_{x} !}{\prod_{a \in F}\left[\left|F_{\leq a}\right|\right]_{x}},
$$

where, for $m \in \mathbb{N},[m]_{x}=\frac{x^{m}-x^{-m}}{x-x^{-1}}$ and $[m]_{x} !=[m]_{x}[m-1]_{x} \ldots[2]_{x}[1]_{x}$.

Theorem 3.3. For any Temperley-Lieb diagram $a: 0 \rightarrow 2 n$ we have an associated forest $F_{a}$, which is simply the poset of arcs, ordered by their nesting. For any Temperley-Lieb diagram $D: t \rightarrow n$, one obtains a unique diagram $\bar{D}: 0 \rightarrow t+n$ by rotating the bottom line clockwise by $\pi$. With this notation, if $\left|q^{2}\right|=\ell$, we have

$$
E_{\ell-1}=\sum_{D} h_{F_{\bar{D}}}(q) D
$$

where the sum is over the diagrams from $\ell-1$ to $\ell-1$, i.e. over the diagram basis of $\mathrm{TL}_{\ell-1}$.

Example 3.4. If $\ell=4$, we may take $q=-\exp \frac{\pi i}{4}$, so that $q^{2}=i$ and the element $E_{3} \in \mathrm{TL}_{3}$ is easily shown to be equal to

$$
E_{3}=1+f_{1} f_{2}+f_{2} f_{1}-\sqrt{2}\left(f_{1}+f_{2}\right) .
$$

Note that our defining parameter for $\mathrm{TL}_{n}$ in this case is $-\left(q+q^{-1}\right)=\sqrt{2}$, and the above element is the familiar one which occurs in the study of the two-dimensional Ising lattice model.

3.2. Properties of the Jones-Wenzl idempotent $E_{\ell-1}$. It is well-known that the Jones-Wenzl idempotent is harmonic. This means that in the Temperley-Lieb category $\mathbf{T}$, if a cap is placed above or below $E_{\ell-1}$, one obtains zero. Diagramatically, this is depicted in Figure 1.

The following result will be needed for the proof of Theorem 7.5 below. 


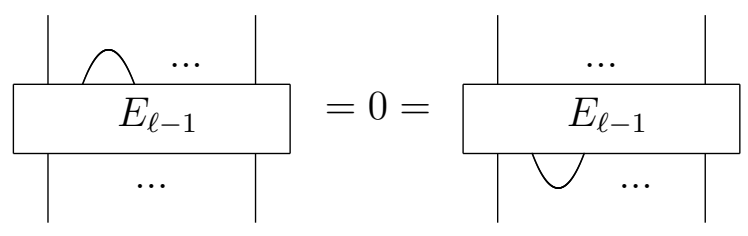

FiguRE 1

Proposition 3.5. (1) Let $\left|q^{2}\right|>n$, and let $E_{i}$ be the Jones-Wenzl idempotent in $\mathrm{TL}_{i}$ for each $i \leq n$. Let $A$ (resp. $U$ ) be the unique diagram from 2 to 0 (resp. 0 to 2) in $\mathbf{T}$, and let $I$ be the unique diagram 1 to 1 . Then

$$
\left(I^{\otimes(n-1)} \otimes A\right)\left(E_{n} \otimes I\right)\left(I^{\otimes(n-1)} \otimes U\right)=\left(\frac{[n-1]_{q}}{[n]_{q}}-[2]_{q}\right) E_{n-1} .
$$

Diagramatically, this equation may be depicted as in Figure 2.

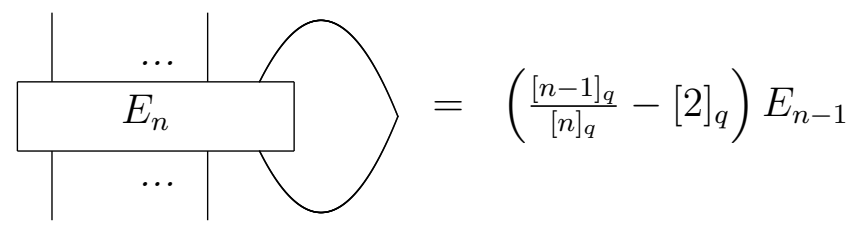

\section{FiguRE 2}

(2) When $\left|q^{2}\right|=\ell,\left(I^{\otimes(\ell-2)} \otimes A\right)\left(E_{\ell-1} \otimes I\right)\left(I^{\otimes(\ell-2)} \otimes U\right)=0$. Diagramatically this is depicted in Figure 3.

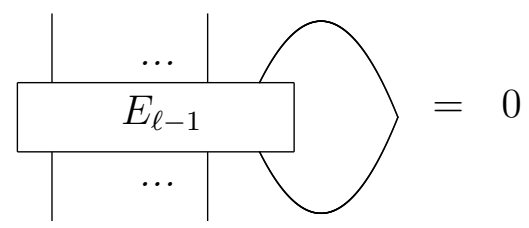

\section{FiguRE 3}

Proof. It is a result of Wenzl (cf. [6, Thm. 3.3, p.461]) that the Jones-Wenzl idempotents satisfy the recursion

$$
E_{n}=E_{n-1} \otimes I+\frac{[n-1]_{q}}{[n]_{q}}\left(E_{n-1} \otimes I\right) f_{n-1}\left(E_{n-1} \otimes I\right)
$$

where $f_{i}$ is the $i^{\text {th }}$ generator of $\mathrm{TL}_{n}$.

Now denote the operator $D \mapsto\left(I^{\otimes(n-1)} \otimes A\right)(D \otimes I)\left(I^{\otimes(n-1)} \otimes U\right)$ by $\gamma: \mathrm{TL}_{n} \rightarrow$ $\mathrm{TL}_{n-1}$. It is easily verified that $\gamma\left(E_{n-1} \otimes I\right)=-[2]_{q} E_{n-1}$ and that $\gamma\left(f_{n-1}\right)=I^{\otimes(n-1)}$. 
Hence

$$
\begin{aligned}
\gamma\left(\left(E_{n-1} \otimes\right.\right. & \left.I) f_{n-1}\left(E_{n-1} \otimes I\right)\right) \\
& =\left(I^{\otimes(n-1)} \otimes A\right)\left(E_{n-1} \otimes I \otimes I\right)\left(f_{n-1} \otimes I\right)\left(E_{n-1} \otimes I \otimes I\right)\left(I^{\otimes(n-1)} \otimes U\right) \\
& =E_{n-1}\left(I^{\otimes(n-1)} \otimes A\right)\left(f_{n-1} \otimes I\right)\left(I^{\otimes(n-1)} \otimes U\right) E_{n-1} \\
& =E_{n-1} \gamma\left(f_{n-1}\right) E_{n-1} \\
& =E_{n-1} .
\end{aligned}
$$

Part (1) now follows immediately by applying the operator $\gamma$ to the relation (3.3).

To obtain the relation in part (2), observe that if $\left|q^{2}\right|=\ell$, then by (1), the left side is equal to $\left(\frac{[\ell-2]_{q}}{[\ell-1]_{q}}-[2]_{q}\right) E_{\ell-2}$. But in our case, $[\ell-1]_{q}=-q^{\ell}$ and $[\ell-2]_{q}=-q^{\ell}[2]_{q}$, which proves $(2)$.

3.3. The Jones quotient. We now wish to consider the quotient of $\mathrm{TL}_{n}$ by the ideal generated by $E_{\ell-1}$.

Definition 3.6. Assume that $\left|q^{2}\right|=\ell$ for a fixed integer $\ell \geq 3$. Let $R_{n}=R_{n}(q)=$ $\left\langle E_{\ell-1}\right\rangle$ be the ideal of $\mathrm{TL}_{n}(q)$ generated by the idempotent $E_{\ell-1} \in \mathrm{TL}_{\ell-1}(q)$, where $\mathrm{TL}_{\ell-1}(q)$ is thought of as a subalgebra of $\mathrm{TL}_{n}(q)$ for $n \geq \ell-1$ in the obvious way. If $n<\ell-1$, we set $R_{n}=0$.

The algebra $Q_{n}=Q_{n}(\ell)(n=\ell-1, \ell, \ell+1, \ldots)$ is defined by

$$
Q_{n}(\ell)=\frac{\mathrm{TL}_{n}}{R_{n}(q)} .
$$

This algebra will be referred to as the "Jones (projection) algebra".

Since we are taking the quotient by the radical of the trace form $\operatorname{tr}_{n}$, it follows that $Q_{n}$ has a non-degenerate invariant trace, and hence that

$$
Q_{n}(\ell) \text { is semisimple. }
$$

Remark 3.7. - The algebras $Q_{n}(\ell)$ are not the maximal semisimple quotients of $\mathrm{TL}_{n}(q)$, as the results of the next section will show.

- We remark that $E_{2}=1-f_{1}$, from which it follows that $Q_{n}(3) \cong \mathbb{C}$ for all $n$.

\section{Representation Theory of $\mathrm{TL}_{n}(q)$.}

We shall apply the basic results of [13] to obtain precise information about the simple modules for $\mathrm{TL}_{n}(q)$ from the general results in $\S 2.3$ about cell modules.

4.1. Review of the representation theory of $\mathrm{TL}_{n}$ at a root of unity. Let $\left|q^{2}\right|=\ell$, where $\ell \geq 3$.

The following description of the composition factors of $W_{t}=W_{t}(n)$ was given in [13, Thm. 5.3], and in the formulation here in [2, Thm. 6.9].

Theorem 4.1. Let $\left|q^{2}\right|=\ell$, fix $n \geq \ell$ and let $\mathcal{T}(n)$ be as above. Let $\mathbb{N}^{\prime}=\{i \in \mathbb{N} \mid$ $i \not \equiv-1(\bmod \ell)\}$. Define $g: \mathbb{N}^{\prime} \rightarrow \mathbb{N}^{\prime}$ as follows: for $t=a \ell+b \in \mathbb{N}^{\prime}, 0 \leq b \leq \ell-2$, define $g(t)=(a+1) \ell+\ell-2-b$. Notice that $g(t)-t=2(\ell-b-1)$, so that $g(t) \geq t+2$ and $g(t) \equiv t(\bmod 2)$. 
(1) For $t \in \mathcal{T}(n) \cap \mathbb{N}^{\prime}$ such that $g(t) \in \mathcal{T}(n)$, there is a non-zero homomorphism $\theta_{t}: W_{g(t)}(n) \rightarrow W_{t}(n)$. These are explicitly described in [13, Thm 5.3], and are the only non-trivial homomorphisms between the cell modules of $\mathrm{TL}_{n}$.

(2) If $t \in \mathcal{T}(n)$ is such that $t \in \mathbb{N}^{\prime}$ and $g(t) \in \mathcal{T}(n)$, then $W_{t}(n)$ has composition factors $L_{t}$ and $L_{g(t)}$, each with multiplicity one. All other cell modules for $\mathrm{TL}_{n}(q)$ are simple.

(3) If $\ell \geq 3$, all the modules $L_{t}(n), t \in \mathcal{T}(n)$, are non-zero, and form a complete set of simple $\mathrm{TL}_{n}(q)$-modules.

Definition 4.2. For $t \in \mathbb{Z}_{\geq 0}$ define functions $w_{t}$ and $l_{t}: \mathbb{N} \rightarrow \mathbb{N}$ by $w_{t}(n)=$ $\operatorname{dim}\left(W_{t}(n)\right)$ and $l_{t}(n)=\operatorname{dim}\left(L_{t}(n)\right)$.

Note that if $t>n, w_{t}(n)=l_{t}(n)=0$. Further $w_{t}(n)=l_{t}(n)=0$ if $n \not \equiv t(\bmod 2)$.

Proposition 4.3. Let $\left|q^{2}\right|=\ell$. We have, for $t \in \mathbb{N}^{\prime}$ (defined as in Theorem 4.1):

$$
l_{t}(n)=\sum_{i=0}^{\infty}(-1)^{i} w_{g^{i}(t)}(n) .
$$

Proof. Note that since $g$ is a strictly increasing function on $\mathbb{N}^{\prime}$, for any particular $n$, the sum on the right side of (4.1) is finite.

It is evident from Theorem $4.1(2)$, that for any $t \in \mathbb{N}^{\prime}$,

$$
l_{t}(n)=w_{t}(n)-l_{g(t)}(n) .
$$

Applying (4.2) with $t$ replaced by $g(t)$ gives $l_{t}(n)=w_{t}(n)-w_{g(t)}(n)+l_{g^{2}(t)}(n)$. Applying this repeatedly, and noting that there is an integer $t_{0} \in \mathbb{N}^{\prime}$ such that $t_{0} \leq n$ and $g\left(t_{0}\right)>n$, we obtain the relation (4.1).

This may be made a little more explicit by the following observation. Fix $\ell=\left|q^{2}\right|$ and $t \geq 0$, write $b(t)=b$, where $t=a \ell+b$, with $0 \leq b \leq \ell-1$. Then for $t \in \mathbb{N}^{\prime}$ we have

$$
\begin{aligned}
g(t) & =t+2 \overline{b(t)} \text { and } \\
g^{2}(t) & =t+2 \ell,
\end{aligned}
$$

where $\overline{b(t)}:=\ell-1-b(t)$.

The equation (4.1) may therefore be written as follows.

Corollary 4.4. We have the following equality of functions on $\mathbb{N}$ :

$$
\begin{aligned}
l_{t} & =\sum_{i=0}^{\infty} w_{t+2 i \ell}-\sum_{i=0}^{\infty} w_{t+2 \overline{b(t)}+2 i \ell} \\
& =\sum_{i=0}^{\infty}\left(w_{t+2 i \ell}-w_{t+2 \overline{b(t)}+2 i \ell}\right) .
\end{aligned}
$$

\section{Generating functions for the Cell modules.}

In this section we recall explicit generating functions for the dimensions of the cell modules of $\mathrm{TL}_{n}(q)$ (cf. [23, Ch. 6]). 


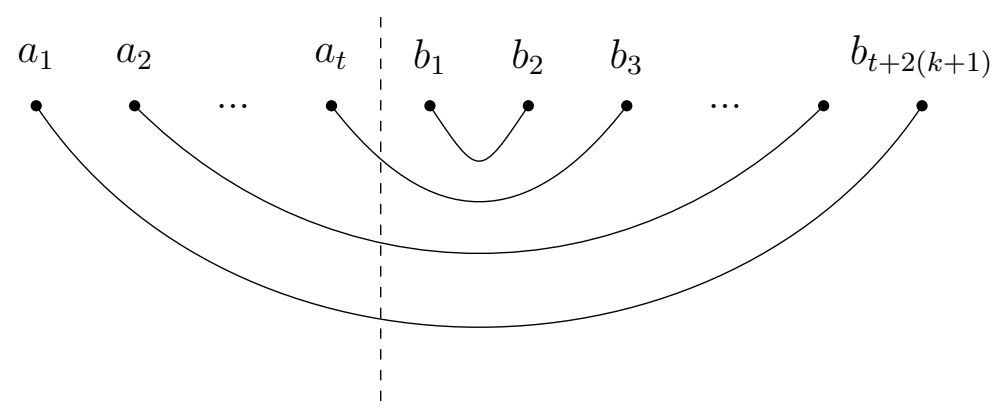

Figure 4. Monic diagram $t \rightarrow t+2(k+1)$ as a diagram $0 \rightarrow 2 t+2(k+1)$.

5.1. Cell modules for $\mathrm{TL}_{n}$. Recall that the cell module $W_{t}(n)$ has a basis consisting of the monic TL-diagrams $D: t \rightarrow n$. Since such diagrams exist only when $t \equiv n(\bmod 2)$, we may write $n=t+2 k, k \geq 0$.

Definition 5.1. For $t, k \geq 0$, we write $w(t, k):=\operatorname{dim} W_{t}(t+2 k)$. By convention, $W_{0}(0)=0$, so that $w(0,0)=0$. Note that by Definition $4.2, w(t, k)=w_{t}(t+2 k)$.

Proposition 5.2. We have the following recursion for $w(t, k)$. For integers $t, k \geq 0$ :

$$
w(t, k+1)=w(t-1, k+1)+w(t+1, k)
$$

Proof. The proof is based on the interpretation of $w(t, k)$ as the number of monic TL-diagrams from $t$ to $t+2 k$.

Consider first the case $t=0$. The assertion is then that $w(0, k+1)=w(1, k)$. But all TL-diagrams $D: 1 \rightarrow 1+2 k$ are monic, as are all diagrams $0 \rightarrow 2 \ell$ (any $\ell$ ). It follows that $w(1, k)=\operatorname{dim}\left(\operatorname{Hom}_{\mathbf{T}}(1,1+2 k)\right)=\operatorname{dim}\left(\operatorname{Hom}_{\mathbf{T}}(0,2+2 k)\right)=w(0, k+1)$. Thus the assertion is true for $t=0$ and all $k \geq 0$. Similarly, if $k=0$, the assertion amounts to $w(t, 1)=w(t-1,1)+w(t+1,0)$. If $t>0$, the left side is easily seen to be equal to $t+1$, while $w(t-1,1)=t$ and $w(t+1,0)=1$. If $t=0$, the left side is equal to $\operatorname{dim}\left(\operatorname{Hom}_{\mathbf{T}}(0,2 k+2)\right)=\operatorname{dim}\left(\operatorname{Hom}_{\mathbf{T}}(1,2 k+1)\right)=w(1, k)$. So the recurrence is valid for $k=0$ and all $t$.

Now consider the general case. Our argument will use the fact that $w(t, k+1)$ may be thought of as the number of TL-diagrams $0 \rightarrow 2 t+2(k+1)$ of the form depicted in Fig. 4, which illustrates the case $k=0$.

The condition that the diagram be monic is simply that each $a_{i}$ is joined to some $b_{j}$, i.e. that each arc crosses the dotted line; of course distinct arcs are nonintersecting.

Evidently such diagrams fall into two types: those in which $\left[a_{t}, b_{1}\right]$ is an arc, and the others. Now the number of diagrams in which $\left[a_{t}, b_{1}\right]$ is an arc is clearly equal to $w(t-1, k+1)$, while those in which $\left[a_{t}, b_{1}\right]$ is not an arc are in bijection with the monic diagrams from $t+1$ to $t+1+2 k$, as is seen by shifting the dotted line one unit to the right. Hence the number of the latter is $w(t+1, k)$, and the recurrence (5.1) is proved.

\subsection{A binomial expression for $w(t, k)$.}

Definition 5.3. For integers $t, k \geq 0$, define

$$
F(t, k)=\left(\begin{array}{c}
t+2 k \\
k
\end{array}\right)-\left(\begin{array}{c}
t+2 k \\
k-1
\end{array}\right) \text {. }
$$


This definition is extended to the domain $\mathbb{Z} \times \mathbb{Z}$ by stipulating that $F(t, k)=0$ if $t<0$ or $k<0$.

It is easily seen that

$$
F(t, k)=\frac{(t+1)(t+2 k)(t+2 k-1) \ldots(t+k+2)}{k !}=\frac{t+1}{t+k+1}\left(\begin{array}{c}
t+2 k \\
k
\end{array}\right),
$$

and that

Lemma 5.4. We have the following recursion for $F(t, k)$. For $t, k \geq 0$ :

$$
F(t, k+1)=F(t-1, k+1)+F(t+1, k) .
$$

5.3. Catalan calculus-generating functions. For $n \geq 0$, write $c(n):=w(0,2 n)$, $c(0)=1$. It is easily seen by inspecting diagrams that for $n>0$,

$$
c(n)=\sum_{k=1}^{n} c(k-1) c(n-k) .
$$

Writing $c(x):=\sum_{n=0}^{\infty} c(n) x^{n}$, the recursion (5.5) translates into

$$
x c(x)^{2}-c(x)+1=0,
$$

from which it is immediate that

$$
c(x)=\frac{1-(1-4 x)^{\frac{1}{2}}}{2 x},
$$

and applying the binomial expansion, that

$$
c(n)=\frac{1}{n+1}\left(\begin{array}{c}
2 n \\
n
\end{array}\right) .
$$

Now define $W_{t}(x)=\sum_{k=0}^{\infty} w(t, k) x^{k}=\sum_{k=0}^{\infty} w_{t}(t+2 k) x^{k}$. Inspection of diagrams shows that the $w(t, k)$ satisfy the following recursion.

$$
w(t, k)=\sum_{\ell=0}^{k} w(t-1, \ell) c(k-\ell),
$$

which translates into the recursion $W_{t}(x)=W_{t-1}(x) c(x)$ for the generating function $W_{t}(x)$. Using the fact that $W_{0}(x)=c(x)$, we have proved Proposition 5.5 below.

Proposition 5.5. For $t=0,1,2, \ldots$, we have $W_{t}(x)=\sum_{k=0}^{\infty} w(t, k) x^{k}=c(x)^{t+1}$.

Corollary 5.6. We have the following equation in $\mathbb{Z}[[x, y]]$.

$$
W(x, y):=\sum_{t, k=0}^{\infty} w(t, k) y^{t} x^{k}=\frac{c(x)}{1-y c(x)} .
$$

5.4. A closed expression for $w(t, k)$. The following result is an easy consequence of the recurrences above.

Theorem 5.7. For integers $t, k \geq 0$, we have

$$
w(t, k)=F(t, k) .
$$

That is,

$$
\operatorname{dim}\left(W_{t}(t+2 k)\right)=\frac{t+1}{t+k+1}\left(\begin{array}{c}
t+2 k \\
k
\end{array}\right)
$$




\section{Generating Functions For the Simple $\mathrm{TL}_{n}(q)$-Modules.}

We assume throughout this section that the order $\left|q^{2}\right|=\ell \in \mathbb{N}$.

Recall (Definition 4.2) that $l_{t}(n)=\operatorname{dim}\left(L_{t}(n)\right)$ is non-zero only if $n=t+2 k$ for some integer $k \geq 0$. For any integer $t \geq 0$ define

$$
L_{t}(x)=L_{t}^{(\ell)}(x)=\sum_{k=0}^{\infty} l_{t}(t+2 k) x^{k} .
$$

In this section we shall give explicit formulae for the power series $L_{t}^{(\ell)}(x)$.

6.1. A recurrence for the functions $l_{t}$. We maintain the following notation, which was introduced in $\S 4$.

Notation. Recall that $\mathbb{N}^{\prime}=\{t \in \mathbb{N} \mid t \not \equiv \ell-1(\bmod \ell)\}$, and for $t \in \mathbb{N}^{\prime}, b(t)=$ $b$, where $t=a \ell+b$ with $0 \leq b \leq \ell-2$. Write $\mathcal{R}=\{0,1,2, \ldots, \ell-2\}, \overline{\mathcal{R}}=$ $\{1,2,3, \ldots, \ell-1\}$ and $b \mapsto \bar{b}$ for the bijection $\mathcal{R} \rightarrow \overline{\mathcal{R}}$ given by $\bar{b}=\ell-1-b$.

Proposition 6.1. Let $t \in \mathbb{N}$ and assume below that $n \equiv t-1(\bmod 2)$.

(1) If $b(t) \in \mathcal{R}$ (i.e. $t \in \mathbb{N}^{\prime}$ ) and $b(t) \neq \ell-2$, then

$$
l_{t}(n+1)=l_{t-1}(n)+l_{t+1}(n) .
$$

(2) For $t \in \mathbb{N}$ with $b(t)=\ell-2$, we have, for $n \equiv t-1(\bmod 2)$,

$$
l_{t}(n+1)=l_{t-1}(n) .
$$

Proof. The relation (5.1) may be written as follows. For all $t, n \in \mathbb{N}$, we have

$$
w_{t}(n+1)=w_{t-1}(n)+w_{t+1}(n) .
$$

Now observe that if $b(t) \in \mathbb{N}^{\prime}$, then applying (6.4) twice, we obtain

$$
w_{t}(n+1)-w_{t+2 \overline{b(t)}}(n+1)=w_{t-1}(n)+w_{t+1}(n)-w_{t+2 \overline{b(t)}-1}(n)-w_{t+2 \overline{b(t)}+1}(n) .
$$

We shall combine the terms of the right side of (6.5) in different ways, depending on the value of $b(t)$. First take $t$ such that $0<t<\ell-2$. Note that $\overline{b(t) \pm 1}=\bar{b}(t) \mp 1$, and for $t$ such that $0<b(t)<\ell-2$ we have $b(t \pm 1)=b(t) \pm 1$. Hence (6.6)

$$
\begin{aligned}
w_{t}(n+1)-w_{t+2 \overline{b(t)}}(n+1) & =\left(w_{t-1}(n)-w_{t+2 \overline{b(t)}+1}(n)\right)+\left(w_{t+1}(n)-w_{t+2 \overline{b(t)}-1}(n)\right) \\
& =\left(w_{t-1}(n)-w_{t-1+2 \overline{b(t)}+2}(n)\right)+\left(w_{t+1}(n)-w_{t+1+2 \bar{t}-2}(n)\right) \\
& =\left(w_{t-1}(n)-w_{t-1+2 \overline{b(t-1)}}(n)\right)+\left(w_{t+1}(n)-w_{t+1+2 \overline{b(t+1)}}(n)\right) .
\end{aligned}
$$

The same relation holds when $t$ in (6.6) is replaced by $t+2 i \ell(i \geq 0)$. That is, for $i \geq 0$ we have

$$
\begin{aligned}
& w_{t+2 i \ell}(n+1)-w_{t+2 i \ell+2 \overline{b(t)}}(n+1)= \\
& \quad\left(w_{t+2 i \ell-1}(n)-w_{t+2 i \ell-1+2 \overline{b(t-1)}}(n)\right)+\left(w_{t+2 i \ell+1}(n)-w_{t+1+2 i \ell+2 \overline{b(t+1)}}(n)\right) .
\end{aligned}
$$

Now given the second line of (4.4), summing both sides of (6.7) over $i \geq 0$ yields the relation $(6.2)$. 
Next take $t \equiv 0(\bmod \ell)$, i.e. $b(t)=0$. Then (6.5) may be written as follows. For $t \equiv 0(\bmod \ell)$, note that $\overline{b(t)}=\ell-1$, and we have

$$
\begin{aligned}
w_{t}(n+1)- & w_{t+2(\ell-1)}(n+1) \\
= & \left(w_{t-1}(n)-w_{t-1+2 \ell}(n)\right)+\left(w_{t+1}(n)-w_{t+1+2(\ell-2)}(n)\right) \\
= & \left(w_{t-1}(n)-w_{t-1+2 \ell}(n)\right)+\left(w_{t+1}(n)-w_{t+1+2(\overline{b(t+1)})}(n)\right) .
\end{aligned}
$$

The same relation (6.8) holds when $t$ is replaced by $t+2 i \ell(i \geq 0)$.

Summing both sides of (6.8) over $i \geq 0$, we see that the first summand on the right is $w_{t-1}(n)$ since all other summands cancel, while the second summand is $l_{t+1}(n)$ by (4.4). Now observe that when $b(t)=0$, then $t-1 \equiv-1(\bmod \ell)$, whence by Theorem 4.1(2), we have $w_{t-1}(n)=\ell_{t-1}(n)$. This completes the proof of $(1)$.

Finally, take $t \equiv \ell-2(\bmod \ell)$, i.e. $b(t)=\ell-2$, so that $\overline{b(t)}=1$ and $g(t)=t+2$. In this case $(6.5)$ reads as follows.

$$
\begin{aligned}
w_{t}(n+1)-w_{t+2}(n+1) & =w_{t-1}(n)+w_{t+1}(n)-\left(w_{t+1}(n)+w_{t+3}(n)\right) \\
& \left.=w_{t-1}(n)-w_{t+3}(n)\right) \\
& \left.=w_{t-1}(n)-w_{t-1+2 \overline{b(t-1)}}(n)\right) .
\end{aligned}
$$

The relation (6.9) remains true when $t$ is replaced by $t+2 i \ell$ for any $i \geq 0$, so that

$$
\left.w_{t+2 i \ell}(n+1)-w_{g(t+2 i \ell)}(n+1)=w_{t+2 i \ell-1}(n)-w_{t+2 i \ell-1+2 \overline{b(t+2 i \ell-1)}}(n)\right),
$$

and summing both sides of $(6.10)$ over $i \equiv \ell-2(\bmod \ell)$ yields the relation $(6.3)$ and completes the proof of the proposition.

6.2. Generating functions. We continue to assume that $q^{2}$ has finite order $\ell$. In this subsection, we give explicit generating functions for the dimensions $l_{t}(n)$ of the simple modules $L_{t}(n)$ of the algebras $\mathrm{TL}_{n}(q)$. Specifically, we give explicit formulae for the power series $L_{t}^{(\ell)}(x)$ defined in (6.1).

Recall (5.7) that $c(x)=1+\sum_{n=1}^{\infty} \frac{1}{n+1}\left(\begin{array}{c}2 n \\ n\end{array}\right) x^{n}$, and define

$$
d(x)=c(x)-1=x c(x)^{2} .
$$

Notice that the relation (5.6) may be written

$$
x(d(x)+1)^{2}=d(x) .
$$

Recall also that for $t \in \mathbb{N}, b(t)$ is defined by $t=a \ell+b(t)$, where $0 \leq b(t) \leq \ell-1$, and that $\mathcal{R}=\{0,1, \ldots, \ell-2\}$.

We shall prove

Theorem 6.2. Maintain the above notation and let $t \in \mathbb{N}$. If $b(t)=\ell-1$, then $L_{t}^{(\ell)}(x)=W_{t}(x)=c(x)^{t+1}$.

If $b(t) \in \mathcal{R}$ then

$$
L_{t}^{(\ell)}(x)=\frac{(d(x)+1)^{t+1}\left(1-d(x)^{\ell-1-b(t)}\right)}{1-d(x)^{\ell}} .
$$

Proof. If $b(t)=\ell-1$ then by Theorem 4.1(2), $W_{t}(n)$ is simple for all $n$. Hence in this case $L_{t}^{(\ell)}(x)=\sum_{k=0}^{\infty} w_{t}(t+2 k) x^{k}=W_{t}(x)=c(x)^{t+1}$ by Proposition 5.5.

Now assume that $b(t) \in \mathcal{R}$. 
It follows from (4.4) that for $k \geq 0$,

$$
\begin{aligned}
l_{t}(t+2 k) & =\sum_{i=0}^{\infty} w_{g^{2 i}(t)}(t+2 k)-\sum_{i=0}^{\infty} w_{g^{2 i+1}(t)}(t+2 k) \\
& =\sum_{i=0}^{\infty} w_{t+2 i \ell}(t+2 k)-\sum_{i=0}^{\infty} w_{t+2 i \ell+2 \overline{b(t)}}(t+2 k) .
\end{aligned}
$$

where $g$ is the function defined in Theorem 4.1 .

Now multiply each side of (6.14) by $x^{k}$ and sum over $k$. We evaluate the two summands separately. We first have

$$
\begin{aligned}
\sum_{k=0}^{\infty} \sum_{i=0}^{\infty} w_{t+2 i \ell}(t+2 k) x^{k} & =\sum_{k=0}^{\infty} \sum_{i=0}^{\infty} w_{t+2 i \ell}(t+2 i \ell+2(k-i \ell)) x^{k} \\
& =\sum_{k=0}^{\infty} \sum_{i=0}^{\infty} w_{t+2 i \ell}(t+2 i \ell+2(k-i \ell)) x^{k-i \ell} x^{i \ell} \\
& =\sum_{i=0}^{\infty} x^{i \ell} \sum_{k=0}^{\infty} w_{t+2 i \ell}(t+2 i \ell+2(k-i \ell)) x^{k-i \ell} \\
& =\sum_{i=0}^{\infty} x^{i \ell} W_{t+2 i \ell}(x) \text { since } w_{t}(n)=0 \text { for } n<t \\
& =\sum_{i=0}^{\infty} x^{i \ell} c(x)^{t+2 i \ell+1} \text { by Proposition } 5.5 \\
& =c(x)^{t+1} \sum_{i=0}^{\infty}\left(x c(x)^{2}\right)^{i \ell} \\
& =\frac{c(x)^{t+1}}{1-\left(x c(x)^{2}\right)^{\ell}} \\
& =\frac{(d(x)+1)^{t+1}}{1-d(x)^{\ell}} \\
&
\end{aligned}
$$

A similar calculation yields that

$$
\sum_{k=0}^{\infty} \sum_{i=0}^{\infty} w_{t+2 i \ell+2 \overline{b(t)}}(t+2 k) x^{k}=\frac{(d(x)+1)^{t+1} d(x)^{\ell-1-b(t)}}{1-d(x)^{\ell}},
$$

and using (6.14), the proof is complete.

6.3. An alternative formula for $L_{t}^{(\ell)}(x)$. We give in this section a formula for $L_{t}^{(\ell)}(x)$ in terms of the polynomials $p_{i}(x)$ defined below.

Definition 6.3. Define a sequence of polynomials $p_{i}(x) \in \mathbb{Z}[x], i=1,2,3, \ldots$ by

$$
\begin{aligned}
p_{1}(x) & =p_{2}(x)=1 \text { and } \\
p_{i+1}(x) & =p_{i}(x)-x p_{i-1}(x) \text { for } i \geq 2 .
\end{aligned}
$$

Thus $p_{3}(x)=1-x, p_{4}(x)=1-2 x, p_{5}(x)=1-3 x+x^{2}$ and $p_{6}(x)=1-4 x+3 x^{2}$, etc. 
Lemma 6.4. Let $y$ be an indeterminate over $\mathbb{Z}$ and $j$ a positive integer.

(1) For each $j \geq 1$ there are unique integers $c_{i}^{j}$ such that

$$
1+y+y^{2}+\cdots+y^{j-1}=\sum_{i=0}^{\left[\frac{j-1}{2}\right]} c_{i}^{j} y^{i}(y+1)^{j-1-2 i} .
$$

(2) The integers $c_{i}^{j}$ satisfy the recurrence $c_{i}^{j+1}=c_{i}^{j}-c_{i-1}^{j-1}$.

(3) We have $\sum_{i=0}^{\left[\frac{j-1}{2}\right]} c_{i}^{j} x^{i}=p_{j}(x)$.

Proof. A polynomial $f(y) \in \mathbb{C}[y]$ is said to be $n$-palindromic $(n \geq 0)$ if $y^{n} f\left(y^{-1}\right)=$ $f(y)$. The $n$-palindromic polynomials form a vector space of dimension $1+\left[\frac{n}{2}\right]$. For fixed $j \geq 1$, the polynomials $y^{i}(y+1)^{j-1-2 i}, 0 \leq i \leq\left[\frac{j-1}{2}\right]$ are all $(j-1)$-palindromic, and since they have leading terms of different degrees, they form a basis of the space of $(j-1)$-palindromic polynomials.

The statement (1) follows easily.

If we write $\sigma_{j}=1+y+y^{2}+\cdots+y^{j-1}$, note that $(1+y) \sigma_{j}=\sigma_{j+1}+y \sigma_{j-1}$. Applying (6.16), we obtain

$$
\sum_{i=0}^{\left[\frac{j-1}{2}\right]} c_{i}^{j} y^{i}(y+1)^{j-2 i}=\sum_{i=0}^{\left[\frac{j}{2}\right]} c_{i}^{j+1} y^{i}(y+1)^{j-2 i}+\sum_{i=0}^{\left[\frac{j-2}{2}\right]} c_{i}^{j-1} y^{i+1}(y+1)^{j-2-2 i} .
$$

Comparing the coefficients of $y^{i}(y+1)^{j-2 i}$ yields the relation (2).

Write $C^{j}(x)=\sum_{i=0}^{\left[\frac{j-1}{2}\right]} c_{i}^{j} x^{i}$. The recurrence (2) shows that $C^{j+1}(x)=C^{j}(x)-$ $x C^{j-1}(x)$. Further, it is easily checked that $C^{1}(x)=C^{2}(x)=1$, which, by comparison with (6.15), completes the proof that $C^{j}(x)=p_{j}(x)$.

Lemma 6.5. Suppose $t=a \ell+b$ with $b=\ell-3$ or $b=\ell-2$. Then

$$
L_{t}^{(\ell)}(x)=\frac{c(x)^{a \ell}}{p_{\ell}(x)}
$$

where $p_{\ell}(x)$ is the polynomial defined in (6.15).

Proof. First observe that by $(6.3), l_{t}(t+2 k)=l_{t-1}(t-1+2 k)$ if $b(t)=\ell-2$, so that $L_{t}^{(\ell)}(x)$ will be the same for the two nominated values of $t$. Now take $t=a \ell+\ell-2$. Applying the formula (6.13), one sees easily that

$$
L_{t}^{(\ell)}(x)=\frac{(d(x)+1)^{a \ell+\ell-1}}{1+d(x)+d(x)^{2}+\cdots+d(x)^{\ell-1}} .
$$

Now using the relation $x(d(x)+1)^{2}=d(x)$ repeatedly, together with Lemma 6.4, one sees that $p_{\ell-1}(x) L_{t}^{(\ell)}(x)=(d(x)+1)^{a \ell}$.

The next result is a generalisation of [18, Thm. 2.3], which deals essentially with the case $0 \leq t \leq \ell-2$ of the Theorem.

Theorem 6.6. With the above notation, we have, for $t=a \ell+b$ with $0 \leq b \leq \ell-2$,

$$
L_{t}^{(\ell)}(x)=\frac{p_{\ell-1-b}(x)}{p_{\ell}(x)} c(x)^{a \ell},
$$

where $c(x)$ is the Catalan series (5.7) and the $p_{i}(x)$ are defined in (6.15). 
Proof. The recurrence (6.2) may be written as follows: for $t$ such that $b(t) \neq 0, \ell-2$, we have $l_{t}(t+2 k)=l_{t-1}(t-1+2 k)+l_{t+1}(t+1+2(k-1))$. Multiplying this relation by $x^{k}$ and summing over $k \geq 0$, we obtain, after rearrangement,

$$
L_{t-1}^{(\ell)}(x)=L_{t}^{(\ell)}(x)-x L_{t+1}^{(\ell)}(x) .
$$

Now fix $a \in \mathbb{N}$ and consider the power series $L_{t}^{(\ell)}(x)$ for $t=a \ell+b, 0 \leq b \leq \ell-2$. We have seen in Lemma 6.5 that when $b=\ell-2$ or $\ell-3$, then $L_{a \ell+\ell-2}^{(\ell)}(x)=$ $L_{a \ell+\ell-3}^{(\ell)}(x)=\frac{c(x)^{a \ell}}{p_{\ell}(x)}$.

Now fix $b$ such that $0 \leq b \leq \ell-3$ and assume that for all $b^{\prime}$ with $\ell-2 \geq b^{\prime} \geq b$, there are polynomials $r_{\ell-1-b^{\prime}}(x)$ such that $L_{a \ell+b^{\prime}}^{(\ell)}(x)=r_{\ell-1-b^{\prime}}(x) L_{a \ell+\ell-2}^{(\ell)}(x)$. Then $r_{1}(x)=r_{2}(x)=1$ and from the recurrence (6.18) we have

$$
\begin{aligned}
L_{a \ell+b-1}^{(\ell)}(x) & =L_{a \ell+b}^{(\ell)}(x)-L_{a \ell+b+1}^{(\ell)}(x) \\
& =r_{\ell-1-b}(x) L_{a \ell+\ell-2}^{(\ell)}(x)-x r_{\ell-2-b}(x) L_{a \ell+\ell-2}^{(\ell)}(x) \\
& =r_{\ell-1-(b-1)}(x) L_{a \ell+\ell-2}^{(\ell)}(x),
\end{aligned}
$$

where $r_{\ell-1-b+1}(x)=r_{\ell-1-b}(x)-x r_{\ell-2-b}(x)$.

It follows that for $b=0,1,2, \ldots, \ell-2, r_{\ell-1-b}(x)=p_{\ell-1-b}(x)$ where $p_{i}(x)$ is as in (6.15), and that $L_{a \ell+b}^{(\ell)}(x)=p_{\ell-1-b}(x) L_{a \ell+\ell-2}^{(\ell)}(x)$. The Theorem now follows by using the expression for $L_{a \ell+\ell-2}^{(\ell)}(x)$ in Lemma 6.5.

Remark 6.7. It is clear that the Jones quotient $Q_{n}(\ell)$ is not generally the largest semisimple quotient of $\mathrm{TL}_{n}(q)$. In fact this is true precisely when $R_{n}$ annihilates all the simple $\mathrm{TL}_{n}$-modules, which happens if and only if $n<\ell$.

For example if $\ell=3, \mathrm{TL}_{3}(q)$ has two simple modules $L_{1}(3)$ and $L_{3}(3)$ of dimension 1 , so its largest semisimple quotient has dimension 2 , while $Q_{3}(3)$ has dimension 1.

Other examples include $\mathrm{TL}_{7}$ where $\ell=5$. We have $\operatorname{dim}\left(Q_{7}\right)=F_{13}=233$ while the maximal semisimple quotient of $\mathrm{TL}_{8}$ has dimension 270 .

6.3.1. Some examples. We give several examples of the application of Theorem 6.6.

(1) When $\ell=3, L_{t}(x)=\frac{c(x)^{3 a}}{1-x}$ for $t=3 a+b, 0 \leq b \leq 1$.

(2) When $\ell=4, L_{1}(x)=L_{2}(x)=\frac{1}{1-2 x}$, while $L_{0}(x)=\frac{p_{3}(x)}{p_{4}(x)}=\frac{1-x}{1-2 x}$. So $\operatorname{dim} L_{0}(2 n)=2^{n-1}, \operatorname{dim} L_{1}(2 n+1)=2^{n}$ and $\operatorname{dim} L_{2}(2 n)=2^{n-1}$.

(3) Take $\ell=5$. We shall determine $L_{i}(x)$ for $i=0,1,2,3$. We have $L_{2}(x)=$ $L_{3}(x)=\frac{1}{1-3 x+x^{2}}, L_{1}(x)=\frac{1-x}{1-3 x+x^{2}}$ and $L_{0}(x)=\frac{1-2 x}{1-3 x+x^{2}}$.

Note that $\frac{L_{0}(x)-1}{x}=L_{1}(x)$.

Let us write

$$
\begin{aligned}
& \frac{1-x}{1-3 x+x^{2}}=\sum_{n=0}^{\infty} a_{n} x^{n} \text { and } \\
& \frac{x}{1-3 x+x^{2}}=\sum_{n=0}^{\infty} b_{n} x^{n} .
\end{aligned}
$$

Then $a_{0}=1, a_{1}=2, a_{2}=5, a_{3}=13$ and $b_{0}=0, b_{1}=1, b_{2}=3, b_{3}=8$.

Let $F_{1}, F_{2}, F_{3}, \cdots=1,1,2,3,5,8,13,21,34, \ldots$ be the Fibonacci sequence. 
We shall show that

$$
a_{0}, b_{1}, a_{1}, b_{2}, a_{2}, b_{3}, a_{3}, \cdots=F_{1}, F_{2}, F_{3}, \ldots,
$$

i.e. that for $i=0,1,2, \ldots$, we have $a_{i}=F_{2 i+1}$ and $b_{i}=F_{2 i}$.

To prove (6.19), given the initial values of the $a_{i}$ and $b_{i}$, it suffices to show that

(i) $b_{n}+a_{n}=b_{n+1}$ for $n \geq 0$, and

(ii) $a_{n}+b_{n+1}=a_{n+1}$ for $n \geq 0$.

For (i), observe that $\sum_{n=0}^{\infty}\left(a_{n}+b_{n}\right) x^{n}=\frac{1-x+x}{1-3 x+x^{2}}=\frac{1}{1-3 x+x^{2}}=\sum_{n=0}^{\infty} b_{n+1} x^{n}$.

Similarly, for (ii), we have $\sum_{n=0}^{\infty}\left(a_{n}+b_{n+1}\right) x^{n}=\frac{2-x}{1-3 x+x^{2}}$, which is readily shown to be equal to $\sum_{n=0}^{\infty} a_{n+1} x^{n}$.

It follows that $L_{3}(x)=L_{2}(x)=\sum_{n=0}^{\infty} b_{n+1} x^{n}$

We have therefore shown that $\operatorname{dim}\left(L_{2}(2+2 n)\right)=\operatorname{dim}\left(L_{3}(3+2 n)\right)=b_{n+1}=$ $F_{2 n+2}, \operatorname{dim}\left(L_{1}(1+2 n)\right)=a_{n}=F_{2 n+1}$ and for $n>0, \operatorname{dim}\left(L_{0}(2 n)\right)=a_{n}-b_{n}=$ $a_{n-1}=F_{2 n-1}$.

(4) Again take $\ell=5$, and consider $L_{8}(x)$. This gives the dimension of the simple $\mathrm{TL}_{n}$-modules $L_{8}(n)$, which are not modules for $Q_{n}$. We have $L_{8}(x)=\frac{c(x)^{5}}{p_{5}(x)}=$ $\frac{1+5 x+20 x^{2}+\ldots}{1-3 x+2 x^{2}}$. So, for example, $\ell_{8}(12)=40$, while $\operatorname{dim}\left(W_{8}(12)\right)=54$. This shows that the latter module has radical of dimension 14.

(5) Take $\ell=6$. We shall compute $L_{t}(x)$ for $t=0,1,2,3,4$. Note first that $L_{3}(x)=L_{4}(x)=\frac{1}{1-4 x+3 x^{2}}$, and since $1-4 x+3 x^{2}=(1-x)(1-3 x)$, we have

$$
L_{3}(x)=L_{4}(x)=\sum_{n=0}^{\infty} \frac{3^{n+1}-1}{2} x^{n}
$$

It follows that $L_{2}(x)=(1-x) L_{3}(x)=\sum_{n=0}^{\infty} 3^{n} x^{n}=\frac{1}{1-3 x}$. Similarly, $L_{1}(x)=$ $\frac{1-2 x}{1-4 x+3 x^{2}}=\sum_{n=0}^{\infty} \frac{3^{n}+1}{2} x^{n}$ and $L_{0}(x)=1+x L_{1}(x)=1+\sum_{n=1}^{\infty} \frac{3^{n-1}+1}{2} x^{n}$.

(6) Take $\ell=7$. An easy but tedious calculation shows that in this case

$$
\begin{aligned}
L_{4}(x)=L_{5}(x) & =1+5 x+19 x^{2}+66 x^{3}+221 x^{4}+728 x^{5}+\ldots \\
L_{3}(x) & =1+4 x+14 x^{2}+47 x^{3}+155 x^{4}+507 x^{5}+\ldots \\
L_{2}(x) & =1+3 x+9 x^{2}+28 x^{3}+89 x^{4}+286 x^{5}+\ldots \\
L_{1}(x) & =1+2 x+5 x^{2}+14 x^{3}+42 x^{4}+131 x^{5}+\ldots \\
L_{0}(x)=1+x L_{1}(x) & =1+x+2 x^{2}+5 x^{3}+14 x^{4}+42 x^{5}+\ldots
\end{aligned}
$$

\section{The Algebras $Q_{n}(\ell)$.}

We assume throughout this section that $\ell$ is fixed and $\left|q^{2}\right|=\ell$. Recall (Definition 3.6) that $Q_{n}(\ell) \simeq \mathrm{TL}_{n}(q) / R_{n}(q)$, where $R_{n}$ is generated by the Jones-Wenzl idempotent $E_{\ell-1} \in \mathrm{TL}_{\ell-1}(q)$. We have seen (3.4) that, although they are not the maximal semisimple quotients of the $\mathrm{TL}_{n}(q)$, the algebras $Q_{n}$ are semisimple and we therefore focus on a description of their simple modules.

7.1. Classification of the simple $Q_{n}(\ell)$-modules. The next statement is elementary. 
Proposition 7.1. Let $n \geq \ell-1$. With $R_{n}$ as above, the simple $Q_{n}$-modules are precisely those simple $\mathrm{TL}_{n}$-modules $L_{t}, t \in \mathcal{T}(n)$, such that $R_{n} L_{t}=0$.

Remark 7.2. If $N$ is a $\mathrm{TL}_{n}$ module, then since $R_{n}=\mathrm{TL}_{n} E_{\ell-1} \mathrm{TL}_{n}$, it follows that $R_{n} N=0$ if and only if $E_{\ell-1} N=0$. Thus the condition in the Proposition is relatively straightforward to check.

Remark 7.3 (Remark concerning notation). Although a priori $E_{\ell-1} \in \mathrm{TL}_{\ell-1}$, we have regarded it as an element of $\mathrm{TL}_{n}$ for any $n \geq \ell-1$. The strictly correct notation for $E_{\ell-1} \in \mathrm{TL}_{n}$, where $n \geq \ell$, is $E_{\ell-1} \otimes I^{\otimes(n-\ell+1)}$, where the tensor product is in the Temperley-Lieb category $\mathbf{T}$, as described in [13] or [21]; that is, it is described diagrammatically as juxtaposition of diagrams, and $I$ is the identity diagram from 1 to 1 . We shall use this notation freely below.

Theorem 7.4. With notation as in Theorem 4.1, let $t \in \mathcal{T}(n)$ satisfy $t \geq \ell-1$. Then the idempotent $E_{\ell-1} \otimes I^{\otimes(n-\ell+1)}$ acts non-trivially on $L_{t}$. Thus $Q_{n}$ has at most $\left[\frac{\ell}{2}\right]$ isomorphism classes of simple modules.

Proof. We begin by showing that if $t \in \mathcal{T}(n)$ and $t \geq \ell-1$ then $\left(E_{\ell-1} \otimes I^{\otimes(n-\ell+1)}\right) W_{t}$ contains all diagrams of the form $I^{\otimes t} \otimes D^{\prime}$, where $D^{\prime}$ is any monic diagram from 0 to $n-t$.

To see this, note that $W_{t}$ is spanned by monic diagrams from $t$ to $n$ in $\mathbf{T}$. Take $D=I^{\otimes t} \otimes D^{\prime} \in W_{t}$, where $D^{\prime}$ is any (monic) diagram from 0 to $n-t$. By the formula in Theorem 3.3, the coefficient of $I^{\otimes(\ell-1)}$ in $E_{\ell-1}$ is 1 . Since all the other summands act trivially on $D$ (because they reduce the number of 'through strings'), it follows that $\left(E_{\ell-1} \otimes I^{\otimes(n-\ell+1)}\right) D=D$ in $W_{t}$, and hence that $D \in\left(E_{\ell-1} \otimes I^{\otimes(n-\ell+1)}\right) W_{t}$.

Now if $D=I^{\otimes t} \otimes D^{\prime}$ as above and $(,)_{t}$ is the canonical bilinear form on $W_{t}$ (see $[12, \S 2])$, then $(D, D)_{t}$ is a power of $-\left(q+q^{-1}\right)$, and hence is non-zero. It follows that $D \notin \operatorname{Rad}_{t}$, and hence that $E_{\ell-1} L_{t} \neq 0$.

It follows from the above result that the only possible simple $Q_{n}$-modules are the $L_{t}$ with $t<\ell-1$.

Theorem 7.5. The simple $Q_{n}$ modules are the $L_{t}$ with $t \leq \ell-2$.

Proof. In view of Proposition 7.1 and Theorem 7.4, it suffices to show that $\left(E_{\ell-1} \otimes\right.$ $\left.I^{\otimes(n-\ell+1)}\right) L_{t}=0$ for $t \leq \ell-2$.

For this, it suffices to show that $\left(E_{\ell-1} \otimes I^{\otimes(n-\ell+1)}\right) W_{t} \subseteq \operatorname{Rad}_{t}$ for $t$ in the relevant range, and this latter statement will follow if we prove that for any two monic diagrams $D_{1}, D_{2} \in W_{t}$, we have

$$
\left(\left(E_{\ell-1} \otimes I^{\otimes(n-\ell+1)}\right) D_{1}, D_{2}\right)_{t}=0 .
$$

To see $(7.1)$, observe that since $\left(E_{\ell-1} \otimes I^{\otimes(n-\ell+1)}\right)$ is an idempotent, it follows from the invariance of the form $(,)_{t}$, that

$$
\left(\left(E_{\ell-1} \otimes I^{\otimes(n-\ell+1)}\right) D_{1}, D_{2}\right)_{t}=\left(\left(E_{\ell-1} \otimes I^{\otimes(n-\ell+1)}\right) D_{1},\left(E_{\ell-1} \otimes I^{\otimes(n-\ell+1)}\right) D_{2}\right)_{t} .
$$

Now for any monic diagram $D \in W_{t},\left(E_{\ell-1} \otimes I^{\otimes(n-\ell+1)}\right) D=0$ unless any upper arc of $D$ whose left end $i \leq \ell-1$ has right end $j \geq \ell$, for otherwise $D$ is annihilated by $E_{\ell-1} \otimes I^{\otimes(n-\ell+1)}$ because $E_{\ell-1}$ is harmonic. It follows, again from the harmonic nature of $E_{\ell-1}$, that if $\left(E_{\ell-1} \otimes I^{\otimes(n-\ell+1)}\right) D \neq 0$, we must have $D=I^{\otimes t_{1}} \otimes D^{\prime} \otimes I^{\otimes t_{2}}$, 
where $t_{1}+t_{2}=t$ and $D^{\prime}: 0 \rightarrow n-t_{1}-t_{2}$ is a diagram such that each of its arcs (it has only upper arcs) has right end in $\{\ell, \ell+1, \ldots, n\}$.

Now let $D_{1}$ and $D_{2}$ be two such diagrams. Then to show that

$$
\left(\left(E_{\ell-1} \otimes I^{\otimes(n-\ell+1)}\right) D_{1},\left(E_{\ell-1} \otimes I^{\otimes(n-\ell+1)}\right) D_{2}\right)_{t}=0,
$$

it suffices to show that $D_{1}^{*}\left(E_{\ell-1} \otimes I^{\otimes(n-\ell+1)}\right) D_{2}=0$.

This latter fact will follow from the harmonic nature of $E_{\ell-1}$, as well as the property of $E_{\ell-1}$ proved in Proposition 3.5(2). To see it, note that if $D_{1}^{*}\left(E_{\ell-1} \otimes\right.$ $\left.I^{\otimes(n-\ell+1)}\right) D_{2}$ is depicted diagramatically in a way similar to Fig. 1,2 or 3, the string emanating from the $(\ell-1)^{\text {st }}$ top vertex (i.e. the rightmost upper vertex of $E_{\ell-1}$ ) either ultimately returns to an upper vertex of $E_{\ell-1}$, in which case we have the zero element by harmonicity, or else it joins the corresponding string emanating from the $(\ell-1)^{\text {st }}$ bottom vertex. In this case we have the zero element by Proposition 3.5(2).

This completes the proof of the theorem.

7.2. Dimensions of the simple $Q_{n}(\ell)$-modules. Since the simple $Q_{n}(\ell)$-modules are just the $L_{t}^{(\ell)}(n) 0 \leq t \leq \ell-2, t \equiv n(\bmod 2)$, their dimensions are given by the formula (6.17). That is,

$$
\sum_{k=0}^{\infty} \operatorname{dim}\left(L_{t}^{(\ell)}(t+2 k)\right) x^{k}=\frac{p_{\ell-1-t}(x)}{p_{\ell}(x)} .
$$

7.3. The case $\ell=4$. Clifford algebras. We have seen in $(6.3 .1)(2)$ that $Q_{2 n+1}(4)$ has just one simple module, whose dimension is $2^{n}$ and that $Q_{2 n}(4)$ has two simple modules, both of dimension $2^{n-1}$. It follows (see also the general formula (8.13)) that $\operatorname{dim} Q_{n}(4)=2^{n-1}$ for $n \geq 1$. We shall see in this section that in this case, $Q_{n}$ is actually the even subalgebra of a Clifford algebra. Because of its connection to the Ising model in statistical mechanics [23], we shall refer to the $Q_{n}(4)$ as the Ising algebras.

Let $U$ be a complex vector space of finite dimension $n$, with a non-degenerate symmetric bilinear form $\langle-,-\rangle$. Then $U$ has an orthonormal basis $u_{1}, \ldots, u_{n}$. If $\gamma_{i}=\frac{1}{\sqrt{2}} u_{i}$ for $i=1, \ldots, n$, then for any $i, j$,

$$
\left\langle\gamma_{i}, \gamma_{j}\right\rangle=\frac{1}{2} \delta_{i, j}
$$

The Clifford algebra $\mathcal{C}_{n}=\mathcal{C}(U,\langle-,-\rangle)$ (for generalities about Clifford algebras we refer the reader to [9]) is defined as

$$
\mathcal{C}_{n}=\frac{T(U)}{I},
$$

where $T(U)=\oplus_{i=0}^{\infty} U^{\otimes i}$ is the free associative $\mathbb{C}$-algebra (or tensor algebra) on $U$, and $I$ is the ideal of $T(U)$ generated by all elements of the form $u \otimes u-\langle u, u\rangle 1$ $(u \in U)$. This last relation may equivalently be written (omitting the $\otimes$ in the multiplication)

$$
u v+v u=2\langle u, v\rangle 1 .
$$

The algebra $\mathcal{C}_{n}$ is evidently generated by any basis of $U$, and hence by (7.3) and (7.5) has the presentation

$$
\left.\mathcal{C}_{n}=\left\langle\gamma_{1}, \ldots, \gamma_{n}\right| \gamma_{i} \gamma_{j}+\gamma_{j} \gamma_{i}=\delta_{i j} \text { for } 1 \leq i, j \leq n\right\rangle .
$$


For any subset $J=\left\{j_{1}<j_{2}<\cdots<j_{p}\right\} \subseteq\{1, \ldots, n\}$, write $\gamma_{J}=\gamma_{j_{1}} \gamma_{j_{2}} \ldots \gamma_{j_{p}}$. It is evident that $\left\{\gamma_{J} \mid J \subseteq\{1,2, \ldots, n\}\right\}$ is a basis of $\mathcal{C}_{n}$ which is therefore $\mathbb{Z}_{2}$-graded (since the relations are in the even subalgebra of the tensor algebra), the even (resp. odd) subspace being spanned by those $\gamma_{J}$ with $|J|$ even (resp. odd).

The following statement is now clear.

Proposition 7.6. The Clifford algebra $\mathcal{C}(U,\langle-,-\rangle)$ has dimension $2^{n}$, where $n=$ $\operatorname{dim}(U)$. Its even subalgebra $\mathcal{C}_{n}^{0}$ has dimension $2^{n-1}$.

The next theorem is the main result of this section; it asserts that the Ising algebra is isomorphic to the even subalgebra of the Clifford algebra.

Theorem 7.7. We continue to assume $\ell=4$ and that $q=-\exp \left(\frac{\pi i}{4}\right)$. Other notation is as above. For $n=3,4, \ldots$ there are surjective homomorphisms $\phi_{n}$ : $\mathrm{TL}_{n}(q) \rightarrow \mathcal{C}_{n}^{0}$ which induce isomorphisms $\bar{\phi}_{n}: Q_{n} \stackrel{\simeq}{\longrightarrow} \mathcal{C}_{n}^{0}$.

Proof. Define $\phi_{n}\left(f_{j}\right)=\frac{1}{\sqrt{2}}\left(1+2 i \gamma_{j} \gamma_{j+1}\right)$. It was remarked by Koo and Saleur $[19, \S 3.1$ eq. (3.2)] (see also [3]) that the $\phi_{n}\left(f_{j}\right)$ satisfy the relations $(2.1)$ in $\mathcal{C}_{n}$, and therefore that $\phi_{n}$ defines a homomorphism from $\mathrm{TL}_{n}$ to $\mathcal{C}_{n}$, and further that $E_{3} \in \operatorname{ker}\left(\phi_{n}\right)$.

It is evident that the image of $\phi_{n}$ is $\mathcal{C}_{n}^{0}$, and therefore that $\bar{\phi}_{n}: Q_{n} \rightarrow \mathcal{C}_{n}^{0}$ is surjective. But by Prop. 7.6 these two algebras have the same dimension, whence $\bar{\phi}_{n}$ is an isomorphism.

7.3.1. Canonical trace. Let $\mathrm{TL}_{n}(q)$ be the $n$-string Temperley-Lieb algebra as above, and assume $\delta:=-\left(q+q^{-1}\right) \neq 0$ is invertible. The canonical Jones trace $\operatorname{tr}_{n}$ on $\mathrm{TL}_{n}(q)$ was defined in (2.2). As pointed out in (3.4), this trace descends to a nondegenerate trace on $Q_{n}$, satisfying similar properties. In the case $\ell=4$ this amounts to the following statement.

Proposition 7.8. There is a canonical trace $\overline{\operatorname{tr}}_{n}$ on $\mathcal{C}_{n}^{0}$, given by taking the constant term (coefficient of 1 ) of any of its elements. This trace corresponds to the Jones trace above in the sense that for $x \in Q_{n}, \operatorname{tr}_{n}(x)=\overline{\operatorname{tr}}_{n}(\phi(x))$. It is therefore nondegenerate.

The proof is easy, and consists in showing that $\overline{\operatorname{tr}}_{n}$ satisfies the analogue of $(2.2)$ in $\mathcal{C}_{n}^{0}$.

7.3.2. The spinor representations of $\mathfrak{s o}(n)$. We give yet another interpretation of the algebra in terms of the spin representations of $\mathfrak{s o}(n)$. Let $\mathrm{SO}(n)$ be the special orthogonal group of the space $(U,\langle-,-\rangle)$ above. Its Lie algebra has basis the set of matrices (with respect to the orthogonal basis $\left.\left(\gamma_{i}\right)\right) J_{i j}:=E_{i j}-E_{j i}, 1 \leq i<$ $j \leq n$, where the $E_{i j}$ are the usual matrix units. This basis of $\mathfrak{s o}(n)$ satisfies the commutation relations

$$
\left[J_{i j}, J_{k l}\right]=\delta_{j k} J_{i l}-\delta_{j l} J_{i k}-\delta_{i k} J_{j l}+\delta_{i l} J_{j k} .
$$

Proposition 7.9. For $n \geq 2$, there are surjective homomorphisms $\psi_{n}: \mathrm{U}(\mathfrak{s o}(n)) \rightarrow$ $\mathcal{C}_{n}^{0} \cong Q_{n}$, such that $\psi_{n}\left(J_{i j}\right)=\omega_{i j}:=\frac{1}{2}\left(\gamma_{i} \gamma_{j}-\gamma_{j} \gamma_{i}\right)$. The irreducible spin representations of $\mathfrak{s o}(n)$ are realised on the simple $Q_{n}$-modules $L_{0}$ and $L_{2}$ when $n$ is even and on $L_{1}$ when $n$ is odd. 
Proof. As this is well known, we give merely a sketch of the argument. To show that $\psi_{n}$ defines a homomorphism, it suffices to observe that the $\omega_{i j}$ satisfy the same commutation relations (7.7) as the $J_{i j}$, and this is straightforward. The surjectivity of $\psi_{n}$ is evident from the observation that $\omega_{i j}=\gamma_{i} \gamma_{j}$, which shows that the image of $\psi_{n}$ contains the whole of $\mathcal{C}_{n}^{0} \simeq Q_{n}$.

\section{The algebras $Q_{n}(\ell)$ And the Reshetikhin-Turaev-Andersen Fusion CATEGORY.}

We show in this section that $Q_{n}(\ell)$ is the endomorphism algebra of a certain truncated tensor product of modules for $\mathrm{U}_{q}=\mathrm{U}_{q}\left(\mathfrak{s l}_{2}\right)$, where $q$ is such that $q^{2}$ is a primitive $\ell^{\text {th }}$ root of unity. An observation about the relevant fusion category permits the determination of the dimension of $Q_{n}(\ell)$. We assume throughout that $\ell \geq 3$.

8.1. Tilting modules for $\mathrm{U}_{q}\left(\mathfrak{s l}_{2}\right)$. For $n \in \mathbb{N}$, let $\Delta_{q}(n)$ be the Weyl module (cf. [2, $\S 1]$ ) of the quantum group $\mathrm{U}_{q}=\mathrm{U}_{q}\left(\mathfrak{s l}_{2}\right)$ and let $T_{q}(n)$ be the unique indecomposable tilting module for $\mathrm{U}_{q}$ with highest weight $n[2, \S 5]$.

It follows from $[2$, Thm. 5.9] that for $n \in \mathbb{N}$,

$$
\Delta_{q}(1)^{\otimes n} \simeq \bigoplus_{t \in \mathbb{N}} l_{t}(n) T_{q}(t)
$$

where $l_{t}(n)=\operatorname{dim}\left(L_{t}(n)\right)$ is the dimension of the simple $\mathrm{TL}_{n}(q)$-module $L_{t}(n)$. Note that $l_{t}(n)$ is non-zero only if $t \equiv n(\bmod 2)$.

Further, the structure of the tilting modules $T_{q}(m)$ is described in [2, Prop. 6.1] as follows.

(1) If $m<\ell$ or $m \equiv-1(\bmod \ell)$, then $T_{q}(m) \simeq \Delta_{q}(m)$ is a simple $\mathrm{U}_{q}$-module.

(2) If $m=a \ell+b$ with $a \geq 1$ and $0 \leq b \leq \ell-2$, then $T_{q}(m)$ has a submodule

isomorphic to $\Delta_{q}(m)$ such that $\frac{T_{q}(m)}{\Delta_{q}(m)} \simeq \Delta_{q}\left(g^{-1}(m)\right)$,

where $g$ is the function defined in (4.3).

8.2. Andersen's fusion category. Andersen proved in [1, Thm. 3.4] a general result for quantised enveloping algebras at a root of unity, which implies in the case of $\mathrm{U}_{q}\left(\mathfrak{s l}_{2}\right)$ that the tilting modules $T_{q}(m)$ with $0 \leq m \leq \ell-2$ are precisely those indecomposable tilting modules with non-zero quantum dimension. This may easily be verified directly in our case using the description (8.2) of the tilting modules. Andersen's result [1, Cor. 4.2] (see also [25]) implies that in our case, we have the following result.

Proposition 8.1. Let $M, N$ be tilting modules for $\mathrm{U}_{q}\left(\mathfrak{s l}_{2}\right)$. Write

$$
M \otimes N=\bigoplus_{n \in \mathbb{N}} m_{n} T_{q}(n)
$$


and define the fusion product $\otimes$ by

$$
M \otimes N=\bigoplus_{n=0}^{\ell-2} m_{n} T_{q}(n) .
$$

Then the fusion product $\underline{\otimes}$ is associative.

This implies that we have a semisimple tensor category $\mathcal{C}_{\text {reg }}$ with objects the tilting modules $\oplus_{n=0}^{\ell-2} m_{n} T_{q}(n)\left(=\oplus_{n=0}^{\ell-2} m_{n} \Delta_{q}(n)\right)\left(m_{n} \in \mathbb{N}\right)$, and tensor product $\underline{\otimes}$.

Definition 8.2. For modules $M=\oplus_{n=0}^{\ell-2} m_{n} T_{q}(n)$ and $M^{\prime}=\oplus_{n=0}^{\ell-2} m_{n}^{\prime} T_{q}(n)$, define

$$
\left(M, M^{\prime}\right)_{\mathrm{U}_{q}}=\operatorname{dim}\left(\operatorname{Hom}_{\mathrm{U}_{q}}\left(M, M^{\prime}\right)\right)=\sum_{n=0}^{\ell-2} m_{n} m_{n}^{\prime} .
$$

Lemma 8.3. Let $M, N \in \mathcal{C}_{\text {red }}$. Then

$$
\left(M \underline{\otimes} \Delta_{q}(1), N\right)_{\mathrm{U}_{q}}=\left(M, N \underline{\otimes} \Delta_{q}(1)\right)_{\mathrm{U}_{q}}
$$

Proof. Since both sides of (8.5) are additive in $M$ and $N$, it suffices to take $M=$ $T_{q}(s)=\Delta_{q}(s)$ and $N=\Delta_{q}(t)$ for $s, t \in\{0,1,2, \ldots, \ell-2\}$. The "reduced ClebschGordan formula" asserts that for $m \in\{0,1,2, \ldots, \ell-2\}$,

$$
\Delta_{q}(m) \underline{\otimes} \Delta_{q}(1) \simeq\left\{\begin{array}{l}
\Delta_{q}(m-1) \oplus \Delta_{q}(m+1) \text { if } m \neq 0 \text { or } \ell-2 \\
\Delta_{q}(1) \text { if } m=0 \\
\Delta_{q}(\ell-3) \text { if } m=\ell-2 .
\end{array}\right.
$$

It is now easily verified that $\operatorname{Hom}_{\mathrm{U}_{q}}\left(M \underline{\otimes} \Delta_{q}(1), N\right) \cong \operatorname{Hom}_{\mathrm{U}_{q}}\left(M, \Delta_{q}(1) \underline{\otimes} N\right)$, and the result follows.

The following result provides an explicit description of the operation $\underline{\otimes}$ in the category $\mathcal{C}_{\text {red }}$.

Proposition 8.4. Suppose $s, t \in \mathbb{Z}$ are such that $0 \leq s, t \leq \ell-2$. Then

$$
\Delta_{q}(s) \underline{\otimes} \Delta_{q}(t) \cong \Delta_{q}(|s-t|) \oplus \Delta_{q}(|s-t|+2) \oplus \cdots \oplus \Delta_{q}(m),
$$

where $m=m(s, t)=\min \{s+t, 2(\ell-2)-(s+t)\}$.

Proof. Note first that by the commutativity of $\underline{\otimes}$, it suffices to prove (8.7) for $s, t$ such that $0 \leq t \leq s \leq \ell-2$. Further, observe that (8.7) holds for $t=0,1$. The case $t=0$ is trivial, while if $t=1(\leq s)$, we have

$$
\Delta_{q}(s) \otimes \Delta_{q}(1) \cong\left\{\begin{array}{l}
\Delta_{q}(s-1) \oplus \Delta_{q}(s+1) \text { if } s<\ell-2 \\
\Delta_{q}(s-1) \text { if } s=\ell-2,
\end{array}\right.
$$

which is precisely the assertion (8.7) in this case. We next show that (8.7) holds when $s=\ell-2$. This assertion amounts to

$$
\Delta_{q}(\ell-2) \underline{\otimes} \Delta_{q}(t) \cong \Delta_{q}(\ell-2-t) \text { for all } t .
$$

We prove (8.8) by induction on $t$; the statement holds for $t=0,1$, as already observed. For $1<t \leq \ell-2$, we have $\Delta_{q}(t-1) \otimes \Delta_{q}(1) \cong \Delta_{q}(t) \oplus \Delta_{q}(t-2)$, whence by induction, $\Delta_{q}(\ell-2) \otimes \Delta_{q}(t-1) \otimes \Delta_{q}(1) \cong \Delta_{q}(\ell-2) \otimes \Delta_{q}(t) \oplus \Delta_{q}(\ell-2) \otimes \Delta_{q}(\ell-2-$ $(t-2))$. But again by induction, the left side is equal to $\Delta_{q}(\ell-2-(t-1)) \underline{\otimes} \Delta_{q}(1) \cong$ $\Delta_{q}(\ell-2-(t-2)) \oplus \Delta_{q}(\ell-2-t)$, which proves $(8.8)$. 
We may therefore now assume that $\ell-3 \geq s \geq t \geq 2$, and proceed by induction on $t$. Using (8.6), and (8.5), we see easily that for any $r$ with $0 \leq r \leq \ell-2$, we have

$$
\begin{aligned}
& \left(\Delta_{q}(s) \underline{\otimes} \Delta_{q}(t), \Delta_{q}(r)\right)_{\mathrm{U}_{q}}= \\
& \quad\left(\Delta_{q}(s) \underline{\otimes} \Delta_{q}(t-1), \Delta_{q}(1) \underline{\otimes} \Delta_{q}(r)\right)_{\mathrm{U}_{q}}-\left(\Delta_{q}(s) \underline{\otimes} \Delta_{q}(t-2), \Delta_{q}(r)\right)_{\mathrm{U}_{q}} .
\end{aligned}
$$

We shall show, using (8.9), that for $0 \leq r \leq \ell-2$,

Assertion 8.5. The multiplicity of $\Delta_{q}(r)$ in both sides of (8.7) is the same.

If $r=0$, the right side of (8.9) is zero unless $s-t+1=1$, i.e. $s=t$, in which case it is 1 . This proves the assertion for $r=0$. If $r=\ell-2$, the first summand on the right side of $(8.9)$ is $\left(\Delta_{q}(s) \otimes \Delta_{q}(t-1), \Delta_{q}(\ell-3)\right)_{\mathrm{U}_{q}}$, which is 1 if $s+t-1=\ell-1$ or $\ell-3$ and zero otherwise. If $s+t-1=\ell-1$, then the second summand on the right side of (8.9) is 1 , whence the right side is zero unless $s+t=\ell-2$, in which case it is 1 . This proves Assertion 8.5 when $r=\ell-2$.

We may therefore assume that $0<r<\ell-2$, so that (8.9) may be written as follows.

$$
\begin{aligned}
\left(\Delta_{q}(s) \underline{\otimes} \Delta_{q}(t), \Delta_{q}(r)\right)_{\mathrm{U}_{q}}=\left(\Delta_{q}(s) \underline{\otimes} \Delta_{q}(t-1), \Delta_{q}(r-1)\right)_{\mathrm{U}_{q}} \\
\quad+\left(\Delta_{q}(s) \underline{\otimes} \Delta_{q}(t-1), \Delta_{q}(r+1)\right)_{\mathrm{U}_{q}}-\left(\Delta_{q}(s) \underline{\otimes} \Delta_{q}(t-2), \Delta_{q}(r)\right)_{\mathrm{U}_{q}} .
\end{aligned}
$$

Now by induction, we have

$$
\Delta_{q}(s) \underline{\otimes} \Delta_{q}(t-1) \cong \Delta_{q}(s-t+1) \oplus \Delta_{q}(s-t+3) \oplus \cdots \oplus \Delta_{q}(m(s, t-1))
$$

and $\Delta_{q}(s) \underline{\otimes} \Delta_{q}(t-2) \cong \Delta_{q}(s-t+2) \oplus \Delta_{q}(s-t+4) \oplus \cdots \oplus \Delta_{q}(m(s, t-2))$.

We consider three cases.

Case 1: $s+t-1>\ell-2$. In this case it is clear that $m(s, t-1)=m(s, t)+1$ and $m(s, t+2)=m(s, t)+2$. Hence in equation (8.10), the last two summands cancel, and we are left with $\left(\Delta_{q}(s) \otimes \Delta_{q}(t), \Delta_{q}(r)\right)_{\mathrm{U}_{q}}=\left(\Delta_{q}(s) \otimes \Delta_{q}(t-1), \Delta_{q}(r-1)\right)_{\mathrm{U}_{q}}$ (for $0<r<\ell-2)$. Bearing in mind that $m(s, t-1)=m(s, t)+1$, this completes the proof of Assertion 8.5 in this case.

Case 2: $s+t-1 \leq \ell-2$ and $s+t \neq \ell-1$. When $s+t-1 \leq \ell-2$, a short calculation shows that $m(s, t-1)=m(s, t)-1$ and $m(s, t-2)=m(s, t)-2$, except in the single case when $s+t=\ell-1$, with which we shall deal separately. We therefore assume for the moment that $s+t \neq \ell-1$, and using (9.7), evaluate each of the three terms in the right side of (8.10). The first term is 1 for $r$ (of the correct parity) such that $s-t+2 \leq r \leq m(s, t)$, and zero otherwise. The second term is 1 for $r$ (of the correct parity) such that $s-t \leq r \leq m(s, t)-2$, and zero otherwise, while the third term is -1 for $r$ (of the correct parity) such that $s-t+2 \leq r \leq m(s, t)-2$, and zero otherwise. This proves Assertion 8.5 in this case.

Case 3: We consider finally the remaining case $s+t=\ell-1$. In this case we have $m(s, t)=\ell-3=m(s, t-2)$, and $m(s, t-1)=\ell-2$. Using this we again evaluate the three terms on the right side of (8.10), recalling that $r \leq \ell-3$. The first term is 1 for $r$ (of the correct parity) such that $s-t+2 \leq r \leq \ell-3$, and zero otherwise. The second term is 1 for $r$ (of the correct parity) such that $s-t \leq r \leq \ell-3$, and zero otherwise while the third term is -1 for $r$ (of the correct parity) such that $s-t+2 \leq r \leq \ell-3$, and zero otherwise.

This completes the proof of Proposition 8.4. 
8.3. Connection with the algebra $Q_{n}(\ell)$. We start with the following observation.

Proposition 8.6. We have

$$
\operatorname{End}_{\mathrm{U}_{q}}\left(\Delta_{q}(1)^{\otimes^{n}}\right) \cong Q_{n}(\ell) .
$$

Proof. It follows from the definition and from (8.1) that

$$
\Delta_{q}(1)^{\otimes n} \simeq \bigoplus_{t=0}^{\ell-2} l_{t}(n) \Delta_{q}(t) .
$$

Since the $\Delta_{q}(t)$ are simple for $0 \leq t \leq \ell-2$, it follows that $\operatorname{End}_{\mathrm{U}_{q}}\left(\Delta_{q}(1) \underline{\otimes}^{n}\right)$ is the direct sum of matrix algebras of degree $l_{t}(n)$, for $t$ such that $0 \leq t \leq \ell-2$ and $t \equiv n(\bmod 2)$. But this latter set of integers is precisely the set of degrees of the simple modules for the semisimple algebra $Q_{n}(\ell)$. The result follows.

Remark 8.7. The results in this paper actually suffice to give a decomposition of $T_{q}(m) \otimes T_{q}(n)$ as a sum of tilting modules. This would give an alternative proof of Proposition 8.4.

Proposition 8.4 may be used to deduce the dimension of $Q_{n}(\ell)$.

Corollary 8.8. (see [11, Thm. 2.9.8]) Define $Q^{(\ell)}(x):=\sum_{n=0}^{\infty} \operatorname{dim}\left(Q_{n+1}(\ell)\right) x^{n}$. Then

$$
Q^{(\ell)}(x)=\frac{p_{\ell-2}(x)}{p_{\ell}(x)}
$$

where the polynomials $p_{i}(x)$ are defined in (6.15).

Proof. It follows from Proposition 8.6 that in the notation of Definition 8.2,

$$
\operatorname{dim}\left(Q_{n+1}(\ell)\right)=\left(\Delta_{q}(1)^{\otimes}(n+1), \Delta_{q}(1)^{\otimes}{ }^{(n+1)}\right)_{\mathrm{U}_{q}}
$$

But by $n$ applications of Lemma 8.3, we see that

$$
\left(\Delta_{q}(1)^{\underline{\otimes}(n+1)}, \Delta_{q}(1)^{\otimes}(n+1)\right)_{\mathrm{U}_{q}}=\left(\Delta_{q}(1)^{\underline{\otimes}(2 n+1)}, \Delta_{q}(1)\right)_{\mathrm{U}_{q}}=l_{1}(2 n+1) .
$$

Finally, by $(7.2)$, we have $\sum_{n=0}^{\infty} l_{1}(2 n+1) x^{n}=\frac{p_{\ell-2}(x)}{p_{\ell}(x)}$, and the proof is complete.

\section{FUSion ALGEBRAS AND FUSION CATEGORIES.}

In this section we investigate some structures which are related to the constructions above. We start with a fusion structure on the representation rings of the algebras $Q_{n}(\ell)$. Throughout this section we take $\ell=\left|q^{2}\right| \geq 3$ as fixed, unless otherwise specified.

9.1. Fusion structure on the Jones algebras. Let $Q_{n}=Q_{n}(\ell)$ be as above. This is a semisimple algebra, and if we write $\mathcal{R}(n):=\{t \in \mathbb{Z} \mid t \equiv n(\bmod 2)$ and $0 \leq t \leq$ $\min \{n, \ell-2\}\}$, then writing $K_{0}$ for the Grothendieck ring,

$$
K_{0}\left(Q_{n}\right) \cong \oplus_{t \in \mathcal{R}(n)} \mathbb{Z}\left[L_{t}(n)\right] .
$$

Define the algebra

$$
K(Q):=\bigoplus_{n \geq 1} K_{0}\left(Q_{n}\right)
$$


where multiplication is given by

$$
\left[L_{s}(m)\right] \circ\left[L_{t}(n)\right]:=\left[\operatorname{Ind}_{Q_{m} \otimes Q_{n}}^{Q_{m+n}}\left(L_{s}(m) \otimes L_{t}(n)\right)\right] .
$$

Remark 9.1. Here $Q_{m} \otimes Q_{n}$ is the subalgebra of $Q_{m+n}$ which is generated by the image of $\mathrm{TL}_{m}(q) \otimes \mathrm{TL}_{n}(q) \subseteq \mathrm{TL}_{m+n}(q)$ under the canonical map $\mathrm{TL}_{m+n}(q) \rightarrow Q_{m+n}(\ell)$. The induced representation $\operatorname{Ind}_{\mathrm{TL}_{m} \otimes \mathrm{TL}_{n}}^{\mathrm{TL}_{m+n}}\left(L_{s}(m) \otimes L_{t}(n)\right)$ may have summands which are not acted upon trivially by $R_{m+n}(q)$. To obtain a representation of $Q_{m+n}$, we consider the submodule of this induced representation of $\mathrm{TL}_{m+n}$ consisting of elements annihilated by $R_{m+n}(q)$.

The multiplication defined above on $K(Q)$ is bilinear, associative and commutative.

Theorem 9.2. We have

$$
\left[L_{s}(m)\right] \circ\left[L_{t}(n)\right]=\sum_{|s-t| \leq r \leq m(s, t)}\left[L_{r}(m+n)\right] .
$$

where $m(s, t)=\min \{s+t, 2(\ell-2)-(s+t)\}$, as in Proposition 8.4.

Proof. It follows from Proposition 8.6 that for $m \geq 1$, as $\mathrm{U}_{q} \otimes Q_{m}$-module,

$$
\Delta_{q}(1)^{\otimes m} \cong \oplus_{s \in \mathcal{R}(m)} \Delta_{q}(s) \otimes L_{s}(m)
$$

It follows that as $\mathrm{U}_{q} \otimes\left(Q_{m} \otimes Q_{n}\right)$-module, we have

$$
\Delta_{q}(1)^{\otimes}(m+n) \cong \bigoplus_{s \in \mathcal{R}(m), t \in \mathcal{R}(n)}\left(\Delta_{q}(s) \underline{\otimes} \Delta_{q}(t)\right) \otimes\left(L_{s}(m) \otimes L_{t}(n)\right) .
$$

But as a module for $\mathrm{U}_{q} \otimes Q_{m+n}$,

$$
\Delta_{q}(1)^{\otimes}(m+n) \cong \bigoplus_{r \in \mathcal{R}(m+n)} \Delta_{q}(r) \otimes L_{r}(m+n) .
$$

Moreover by Proposition 8.4, we may expand (9.4) as follows.

$$
\left(\Delta_{q}(s) \underline{\otimes} \Delta_{q}(t)\right) \otimes\left(L_{s}(m) \otimes L_{t}(n)\right) \cong \bigoplus_{r \in \mathcal{R}(m+n),|s-t| \leq r \leq m(s, t)} \Delta_{q}(r) \otimes\left(L_{s}(m) \otimes L_{t}(n)\right) .
$$

Comparing this last equation with (9.5), we see that given $r \in \mathcal{R}(m+n)$, we have

$$
\operatorname{Res}_{Q_{m} \otimes Q_{n}}^{Q_{m+n}}\left(L_{r}(m+n)\right) \cong \bigoplus_{|s-t| \leq r \leq m(s, t)}\left(L_{s}(m) \otimes L_{t}(n)\right)
$$

But by Frobenius reciprocity, the multiplicity of $L_{s}(m) \otimes L_{t}(n)$ in $\operatorname{Res}_{Q_{m} \otimes Q_{n}}^{Q_{m+n}}\left(L_{r}(m+\right.$ $n))$ is equal to that of $L_{r}(m+n)$ in $\operatorname{Ind}_{Q_{m} \otimes Q_{n}}^{Q_{m+n}}\left(L_{s}(m) \otimes L_{t}(n)\right)$. It follows that (9.6) is equivalent to:

$$
\operatorname{Ind}_{Q_{m} \otimes Q_{n}}^{Q_{m+n}}\left(L_{s}(m) \otimes L_{t}(n)\right) \cong \bigoplus_{r:|s-t| \leq r \leq m(s, t)} L_{r}(m+n),
$$

which is the required statement. 
9.2. Connections with the Virasoro algebra. We conclude with some observations and speculations about possible connections of our results with Virasoro algebras. Recall that the Virasoro algebra $\mathcal{L}=\oplus_{i \in \mathbb{Z}} \mathbb{C} L_{i} \oplus \mathbb{C} C$ has irreducible highest weight modules $L(c, h)$ with highest weight $(c, h)$, where $c, h(\in \mathbb{C})$ are respectively the central charge and the eigenvalue of $L_{0}$. It was conjectured by Friedan, Qiu and Schenker [7] that $L(c, h)$ is unitarisable if and only if either

(1) $c \geq 1$ and $h \geq 0$, or

(2) there exist integers $m \geq 2, r$ and $s$ with $0<r<m$ and $0<s<m+1$ such that

$$
c=c_{m}:=1-\frac{6}{m(m+1)} \text { and } h=h_{r, s}:=\frac{((m+1) r-m s)^{2}-1}{4 m(m+1)} .
$$

As $h_{r, s}=h_{m-r, m+1-s}$, it is suffices to take, $1 \leq s \leq r<m$ in the latter case. The "if" part of this statement was proved by Goddard, Kent and Olive [10] and the "only if" part was proved by Langlands [20].

This result bears a superficial resemblance to Jones' result on the range of values of the index of a subfactor as was mentioned in the preamble. Thus it might be expected that case (2) is somehow connected with our algebras $Q_{n}(\ell)$ for $\ell=3,4,5, \ldots$

Further, there are several instances in the literature (see, e.g. [8, 19, 24]) which hint at a connection between $Q_{n}(\ell)$ and the minimal unitary series of $\mathcal{L}$ with central charge $c_{\ell}$. Our work may provide some further evidence along those lines.

For $\ell=3, c=0$, and there is just one irreducible representation, viz. the trivial one. This is 'consistent' with $Q_{n}(3)=\mathbb{C}$. For $\ell=4, c=\frac{1}{2}$. This case is the Ising model, or equivalently, the 2-state Potts model, as we have already observed.

Now the abelian groups $K_{0}\left(Q_{2 n}\right) n=1,2,3, \ldots$ form an inverse system, as do the $K_{0}\left(Q_{2 n+1}\right)$, via the maps $\left[L_{t}(n+2)\right] \mapsto\left\{\begin{array}{l}{\left[L_{t}(n)\right] \text { if } n-t \in 2 \mathbb{Z}_{\geq 0}} \\ 0 \text { otherwise }\end{array}\right.$.

Define the abelian groups $K\left(Q_{\text {even }}(\ell)\right):=\lim \left(K\left(Q_{2 n}(\ell)\right)\right)$ and $K\left(Q_{\text {odd }}(\ell)\right):=$ $\lim _{\leftarrow}\left(K\left(Q_{2 n+1}(\ell)\right)\right)$. Then $K\left(Q_{\infty}\right):=K\left(Q_{\text {even }}(\ell)\right)^{\leftarrow} \oplus K\left(Q_{\text {odd }}(\ell)\right)$ has a $\mathbb{Z}$-basis which may be written $\left\{\left[L_{t}\right] \mid t=0,1,2, \ldots, \ell-2\right\}$. Define a multiplication on $K\left(Q_{\infty}\right)$ by

$$
\left[L_{s}\right] \circ\left[L_{t}\right]=\sum_{\substack{r \equiv s+t(2) \\|s-t| \leq r \leq m(s, t)}}\left[L_{r}\right]
$$

By the usual properties of inverse limits, we have maps $\tau_{n}: K\left(Q_{\infty}\right) \longrightarrow K_{0}\left(Q_{n}(\ell)\right)$, given by

$$
\tau_{n}\left(\left[L_{t}\right]\right)=\left\{\begin{array}{l}
{\left[L_{t}(n)\right] \text { if } n-t \in 2 \mathbb{Z}_{\geq 0}} \\
0 \text { otherwise }
\end{array}\right.
$$

Theorem 9.2 implies that the ring $K\left(Q_{\infty}\right)$ is a 'stable limit' or 'completion' of the Grothendieck ring $K(Q)\left(=\bigoplus_{n=1}^{\infty} K_{0}\left(Q_{n}(\ell)\right)\right)$ in the sense that for all $m, n, s$ and $t$,

$$
\tau_{m}\left(\left[L_{s}\right]\right) \circ \tau_{n}\left(\left[L_{t}\right]\right)=\tau_{m+n}\left(\left[L_{s}\right] \circ\left[L_{t}\right]\right) .
$$

Moreover the ring $K\left(Q_{\infty}\right)$ is isomorphic [27, (4.6), p.369] to the fusion ring of $\widehat{\mathfrak{s l}}_{2}$ at level $\ell-2$, which in turn is isomorphic to the subring of the fusion algebra of $\mathcal{L}$ with central charge $c_{\ell-1}$ generated by $\left[L\left(c_{l-1}, h_{1, s}\right)\right](1 \leq s \leq l-1)($ cf. $[14, \S 9.3])$. 


\section{REFERENCES}

[1] Henning Haahr Andersen, "Tensor products of quantized tilting modules", Comm. Math. Phys. 149 (1992), no. 1, 149-159.

[2] Henning H. Andersen, Gustav I. Lehrer, and Ruibin Zhang, "Cellularity of certain quantum endomorphism algebras", Pacific J. Math. 279 (2015), no. 1-2, 11-35.

[3] Connes, Alain and Evans, David E., "Embedding of $U(1)$-current algebras in noncommutative algebras of classical statistical mechanics", Comm. Math. Phys. 121 (1989), no. 3, $507-525$.

[4] Curtis, Charles W. and Reiner, Irving, "Representation theory of finite groups and associative algebras", Pure and Applied Mathematics, Vol. XI Interscience Publishers, a division of John Wiley \& Sons, New York-London 1962 xiv+ 685 pp.

[5] Erdmann, Karin, "Tensor products and dimensions of simple modules for symmetric groups", Manuscripta Math. 88 (1995), no. 3, 357-386.

[6] Frenkel, Igor B. and Khovanov, Mikhail G., "Canonical bases in tensor products and graphical calculus for $\mathrm{U}_{q}\left(\mathfrak{s l}_{2}\right)$, Duke Math. J. 87 (1997), no. 3, 409-480.

[7] Friedan D., Qiu D. and Shenker S., "Conformal Invariance, Unitarity and Critical Exponents in Two Dimensions", Phys. Rev. Lett. 52 (1984), 1575-1578.

[8] Gainutdinov A. and Saleur H., "Fusion and braiding in finite and affine Temperley-Lie categories", preprint, arXiv:1606.04530v1

[9] Garling, D. J. H., "Clifford algebras: an introduction", London Mathematical Society Student Texts, 78 Cambridge University Press, Cambridge, 2011.

[10] Goddard P, Kent A. and Olive D., "Unitary representations of the Virasoro and uperVirasoro Algebras", Commun. Math. Phys. 103 (1986), 105-119.

[11] Goodman, Frederick M., de la Harpe, Pierre and Jones, Vaughan F. R. " Coxeter graphs and towers of algebras" Mathematical Sciences Research Institute Publications, 14 SpringerVerlag, New York, 1989. x+288 pp. ISBN: 0-387-96979-9

[12] Graham, J. J. and Lehrer, G. I. "Cellular algebras", Invent. Math. 123 (1996), no. 1, 1-34.

[13] Graham, J. J. and Lehrer, G. I., " The representation theory of affine Temperley-Lieb algebras", Enseign. Math. (2) 44 (1998), no. 3-4, 173-218.

[14] Iohara K. and Koga Y., Representation Theory of the Virasoro Algebra, Springer Monograph in Math., Springer, 2011.

[15] Jones, V. F. R., "Index for subfactors", Invent. Math. 72 (1983), no. 1, 1-25.

[16] Jones, V. F. R., "Hecke algebra representations of braid groups and link polynomials", Ann. of Math. (2) 126 (1987), no. 2, 335-388.

[17] Jones, Vaughan F. R., "A polynomial invariant for knots via von Neumann algebras", Bull. Amer. Math. Soc. (N.S.) 12 (1985), no. 1, 103-111.

[18] Jones, Vaughan F. R. and Reznikoff, Sarah A., "Hilbert space representations of the annular Temperley-Lieb algebra", Pacific J. Math. 228 (2006), no. 2, 219-249.

[19] Koo, W. M. and Saleur, H, "Representations of the Virasoro algebra from lattice models", Nuclear Phys. B 426 (1994), no. 3, 459-504.

[20] Langlands R., "On unitary representations of the Virasoro algebra", in Infinite-dimensional Lie algebras and their applications (Montreal 1986), World Sci. Publ., 1988, 141-159.

[21] G. I. Lehrer and R. B. Zhang, "The Brauer Category and Invariant Theory", J. Eur. Math. Soc. (JEMS) 17 (2015), no. 9, 2311-2351.

[22] Martin, P. P., Launer, G. and Westbury, B. W., "The Potts models and a generalisation of the Clifford algebras", Bull. London Math. Soc. 21 (1989), no. 6, 544-550.

[23] Martin, Paul, "Potts models and related problems in statistical mechanics", Series on Advances in Statistical Mechanics, 5. World Scientific Publishing Co., Inc., Teaneck, NJ, 1991. xiv+344 pp. ISBN: 981-02-0075-7

[24] Nichols, A., "The Temperley-Lieb algebra and its generalizations in the Potts and $X X Z$ models, Jour. Stat. Mech. (2006), 46 pages.

[25] N. Reshetikhin and V.G. Turaev, "Invariants of 3-manifolds via link polynomials and quantum groups", Invent. Math. 103 (1991), no. 3, 547-597. 
[26] Reznikoff, Sarah A., "Temperley-Lieb planar algebra modules arising from the ADE planar algebras", J. Funct. Anal. 228 (2005), no. 2, 445-468.

[27] Verlinde, Erik, "Fusion rules and modular transformations in 2D conformal field theory", Nuclear Phys. B 300 (1988), no. 3, 360-376.

[28] Wenzl, Hans, "On sequences of projections" C. R. Math. Rep. Acad. Sci. Canada 9 (1987), no. $1,5-9$.

Univ Lyon, Université Claude Bernard Lyon 1, CNRS UMR 5208, Institut Camille Jordan, 43 Boulevard du 11 Novembre 1918, F-69622 Villeurbanne Cedex, France

E-mail address: iohara@math.univ-lyon1.fr

School of Mathematics and Statistics, University of Sydney, NSW 2006, AusTRALIA

E-mail address: gustav.lehrer@sydney.edu.au, ruibin.zhang@sydney.edu.au 